\title{
Analysis of Observer Performance in Known-Location Tasks for Tomographic Image Reconstruction
}

\author{
Anastasia Yendiki*, Student Member, IEEE, and Jeffrey A. Fessler, Fellow, IEEE
}

\begin{abstract}
We consider the task of detecting a statistically varying signal of known location on a statistically varying background in a reconstructed tomographic image. We analyze the performance of linear observer models in this task. We show that, if one chooses a suitable reconstruction method, a broad family of linear observers can exactly achieve the optimal detection performance attainable with any combination of a linear observer and linear reconstructor. This conclusion encompasses several well-known observer models from the literature, including models with a frequency-selective channel mechanism and certain types of internal noise. Interestingly, the "optimal" reconstruction methods are unregularized and in some cases quite unconventional. These results suggest that, for the purposes of designing regularized reconstruction methods that optimize lesion detectability, known-location tasks are of limited use.
\end{abstract}

Index Terms-Emission tomography, lesion detection, observer models, channelized Hotelling observer, penalized maximum-likelihood.

\section{INTRODUCTION}

O BJECTIVE evaluation of the quality of an image requires specifying the goal that the image will be used to achieve. Typical uses of medical images can be categorized into estimation, where the goal is to measure the value of some parameter of interest (e.g., radioactivity distribution), and classification, where the goal is to decide among several possible states of the truth (e.g., the presence or absence of a tumor) [1]. By evaluating images with respect to a task of interest, one can compare and rank the imaging systems or reconstruction algorithms that produce these images. In the case of classification tasks, such evaluations can be performed directly by applying ROC analysis to experiments that record the classification performance of human observers [2]-[4]. However, not only are such experiments time-consuming, but also the performance of human observers does not lend itself to optimization through analytical tools. The alternative is to turn to mathematical observer models.

Manuscript received January 24, 2005; revised June 21, 2005. This work was supported in part by the National Science Foundation (NSF) under Grant BES9982349 and NCI Grant P01 CA87634. The Associate Editor responsible for coordinating the review of this paper and recommending its publication was E. Krupinski. Asterisk indicates corresponding author.

*A. Yendiki was with the University of Michigan, Ann Arbor, MI 48109-2122, USA. She is now with the HMS/MGH/MIT Martinos Center for Biomedical Imaging, Charlestown, MA 02129 USA (e-mail: ayendiki@nmr.mgh.harvard.edu).

J. A. Fessler is with the University of Michigan, Ann Arbor, MI 48109-2122 USA.

Digital Object Identifier 10.1109/TMI.2005.859714
The ideal observer models that are well-known from statistical decision theory [5] exploit all available statistical information on the classification task at hand. However, researchers have found that human observers do not perform ideally and have proposed observer models that account for human suboptimality [6]-[9]. As a result, various popular linear observer models exist in the literature, either in their ideal form [10], or with the addition of frequency-selective channels and internal noise [11, Section 14.2.2]. The channelized Hotelling observer (CHO) [9] in particular, has been found to predict human observer performance in several detection tasks [12]-[17]. Furthermore, strategies have been proposed for estimating the template of a linear observer model directly from human observer data [18], [19].

Since the performance of the ideal observer is invariant to any nonsingular data transformation, it cannot be used to evaluate and rank image reconstruction methods. Therefore, one can optimize reconstruction methods with respect to detectability only for suboptimal observer models such as the ones mentioned above. Significant attention has been focused on analyzing the performance of several such linear observers for the task of detecting a signal of known location in tomographic images [20]-[25]. One can use these analytical performance approximations to tune user-specified parameters involved in image reconstruction methods, such as regularization parameters in penalized-likelihood reconstruction [26].

In this paper, we extend our analysis from [22] to show that a broad family of linear observer models and their channelized counterparts can exactly achieve optimal performance (for a certain internal noise model) in the detection of a statistically varying signal on a statistically varying background without the need for regularization. Further insight into the performance of channelized observers can be gained through local shift invariance analysis. Using such an analysis, we present evidence of more situations where the $\mathrm{CHO}$ in particular can achieve approximately optimal performance in this task without regularization.

The paper is structured as follows. Section II defines the detection task and the general form of the observers and reconstruction methods that we will be considering. Section III analyzes the detection performance of several well-known observers, with the objective of finding reconstruction methods that allow each of these observers to achieve optimal performance. We show that there is a broad family of linear observers that can achieve the optimum with unregularized reconstruction. Section IV summarizes the conclusions of our analysis. 


\section{THE DETECTION TASK}

\section{A. Object Model}

Let $\boldsymbol{f}$ denote the true object being imaged (or an approximation of the true object in $\mathbb{R}^{n_{\mathrm{p}}}$ ). To express our uncertainty about the object, we allow it to be a random process parameterized by two spatial coordinates. The object $\boldsymbol{f}$ consists of a background $\boldsymbol{f}_{b}$ and it may or may not also contain a signal of interest $\boldsymbol{f}_{s}$, which we assume to be localized in space. The detection task is to determine whether the signal $f_{s}$ is present or not in the object $f$, i.e., to decide between the following pair of hypotheses:

$$
\begin{aligned}
& H_{0}: f=f_{b} \quad \text { (signal absent) } \\
& H_{1}: f=f_{b}+\boldsymbol{f}_{s} \quad \text { (signal present). }
\end{aligned}
$$

In emission tomography, for example, where the object $f$ is a radioactivity distribution, the background $f_{b}$ could correspond to normal radioactivity and the signal $f_{s}$ to additional radioactivity absorbed in the area of a lesion.

The background $\boldsymbol{f}_{b}$ and the signal $\boldsymbol{f}_{s}$ are random and statistically independent. We denote their expectations by $\overline{\boldsymbol{f}}_{b}$ and $\overline{\boldsymbol{f}}_{s}$, respectively. We denote their covariances by $\mathcal{K}_{b}$ and $\mathcal{K}_{s}$, respectively. In the general case where both background and signal are random, $\mathcal{K}_{b}$ and $\mathcal{K}_{s}$ are positive definite. In the special case known as the signal known exactly (SKE) detection task, we have $\mathcal{K}_{s}=\mathbf{0}$ and, thus, a deterministic signal $\boldsymbol{f}_{s}=\overline{\boldsymbol{f}}_{s}$. Similarly, in the background known exactly (BKE) task, we have $\mathcal{K}_{b}=\mathbf{0}$ and, thus, a deterministic background $\boldsymbol{f}_{b}=\bar{f}_{b}$. In all cases, we assume that $\bar{f}_{b}, \bar{f}_{s}, \mathcal{K}_{b}$, and $\mathcal{K}_{s}$ are known.

\section{B. Measurement Model}

In the applications that interest us, the true object $\boldsymbol{f}$ cannot be observed directly. The decision between hypotheses $H_{0}$ and $H_{1}$ has to be based on a noisy measurement $\boldsymbol{y} \in \mathbb{R}^{n_{\mathrm{d}}}$ that depends on $\boldsymbol{f}$. For a given instance of the object $\boldsymbol{f}$, the measurement $\boldsymbol{y}$ is random due to noise inherent in the imaging system.

In the following, we use $\mathrm{E}[\cdot]$ to denote expectation and $\operatorname{Cov}\{\cdot\}$ to denote covariance. All moments are averages over both $\boldsymbol{y}$ and $f$, except when they are accompanied by the subscript $f$, in which case they are averages only over $f$, or when they are conditional averages over $\boldsymbol{y}$ for a given instance of $\boldsymbol{f}$, in which case the usual $\cdot \mid \boldsymbol{f}$ notation is used. Finally, we use the notation $\cdot \mid H_{i}$ when referring to moments conditional on the event that hypothesis $H_{i}$ is true. We assume knowledge of the following moments of the measurement $y$ :

$$
\begin{gathered}
\overline{\boldsymbol{y}} \triangleq \mathrm{E}\left[\boldsymbol{y} \mid H_{1}\right]-\mathrm{E}\left[\boldsymbol{y} \mid H_{0}\right] \\
\boldsymbol{\Pi}_{i} \triangleq \operatorname{Cov}\left\{\boldsymbol{y} \mid H_{i}\right\}, \quad i=0,1
\end{gathered}
$$

where we use " $\triangleq "$ " to denote definitions.

In emission tomography, the measurement $\boldsymbol{y}$ is a noisy sinogram, whose elements are independent and Poisson-distributed conditional on the object $f$. The conditional mean and covariance of the sinogram $\boldsymbol{y}$ are, respectively

$$
\begin{aligned}
\mathrm{E}[\boldsymbol{y} \mid \boldsymbol{f}] & =\mathcal{A} \boldsymbol{f}+\boldsymbol{r} \\
\operatorname{Cov}[\boldsymbol{y} \mid \boldsymbol{f}] & =\operatorname{diag}\{\mathcal{A} \boldsymbol{f}+\boldsymbol{r}\}
\end{aligned}
$$

where we use the notation $\operatorname{diag}\{\boldsymbol{v}\}$ for a diagonal matrix with diagonal elements equal to the elements of vector $v$. The linear operator $\mathcal{A}$ models the tomographic imaging system and the vector $\boldsymbol{r} \in \mathbb{R}^{n_{\mathrm{d}}}$ represents "background events" such as scatter and/or random coincidences [27], [28]. Both $\mathcal{A}$ and $\boldsymbol{r}$ are assumed to be deterministic and known. The moments of the sinogram $\boldsymbol{y}$ under hypothesis $H_{i}, i=0,1$ are then given by

$$
\begin{aligned}
\mathrm{E}\left[\boldsymbol{y} \mid H_{i}\right]= & \mathrm{E}_{\boldsymbol{f}}\left[\mathrm{E}[\boldsymbol{y} \mid \boldsymbol{f}] \mid H_{i}\right]=\mathcal{A E}_{\boldsymbol{f}}\left[\boldsymbol{f} \mid H_{i}\right]+\boldsymbol{r} \\
\operatorname{Cov}\left\{\boldsymbol{y} \mid H_{i}\right\}= & \mathrm{E}_{\boldsymbol{f}}\left[\operatorname{Cov}\{\boldsymbol{y} \mid \boldsymbol{f}\} \mid H_{i}\right]+\operatorname{Cov}_{\boldsymbol{f}}\left\{\mathrm{E}[\boldsymbol{y} \mid \boldsymbol{f}] \mid H_{i}\right\} \\
= & \operatorname{diag}\left\{\mathcal{A} \mathrm{E}_{\boldsymbol{f}}\left[\boldsymbol{f} \mid H_{i}\right]+\boldsymbol{r}\right\} \\
& +\mathcal{A C o v}_{\boldsymbol{f}}\left\{\boldsymbol{f} \mid H_{i}\right\} \mathcal{A}^{\prime}
\end{aligned}
$$

where we use " " " to denote the adjoint of an operator or equivalently the complex transpose of a matrix. Under the assumption that $f_{b}$ and $f_{s}$ are independent, using (4) and (5) for each of the two hypotheses in (1) and substituting in (2) and (3) yields, for emission tomography

$$
\begin{aligned}
\overline{\boldsymbol{y}} & =\boldsymbol{\mathcal { A }} \overline{\boldsymbol{f}}_{s} \\
\Pi_{0} & =\operatorname{diag}\left\{\mathcal{A} \overline{\boldsymbol{f}}_{b}+\boldsymbol{r}\right\}+\mathcal{A} \mathcal{K}_{b} \mathcal{A}^{\prime} \\
\Pi_{1} & =\Pi_{0}+\operatorname{diag}\left\{\mathcal{A} \overline{\boldsymbol{f}}_{s}\right\}+\mathcal{A} \mathcal{K}_{s} \mathcal{A}^{\prime} .
\end{aligned}
$$

\section{Image Reconstruction Methods}

An image reconstruction method is a mapping of the measurements $\boldsymbol{y}$ into an estimated image $\hat{f}$. We focus here on linear reconstruction methods, as several common tomographic reconstruction techniques either are or can be approximated as linear. We denote a generic linear reconstructor by an operator $\mathcal{Z}$. The reconstructed image is then given by

$$
\hat{\boldsymbol{f}}(\boldsymbol{y})=\mathcal{Z} \boldsymbol{y} .
$$

We may view the reconstruction $\hat{f}$ either as a vector in a Hilbert space, in which case $\mathcal{Z}$ is a general linear mapping from $\mathbb{R}^{n_{\mathrm{d}}}$ to that Hilbert space, or as a discrete representation in $\mathbb{R}^{n_{\mathrm{p}}}$, in which case $\mathcal{Z}$ is a matrix in $\mathbb{R}^{n_{\mathrm{p}} \times n_{\mathrm{d}}}$. Combining the linear reconstruction method in (9) with the data moments from (2) and (3) yields the following expressions for the moments of the reconstructed image:

$$
\begin{aligned}
\mathrm{E}\left[\hat{\boldsymbol{f}} \mid H_{1}\right]-\mathrm{E}\left[\hat{\boldsymbol{f}} \mid H_{0}\right] & =\mathcal{Z} \overline{\boldsymbol{y}} \\
\operatorname{Cov}\left\{\hat{\boldsymbol{f}} \mid H_{i}\right\} & =\mathcal{Z} \boldsymbol{\Pi}_{i} \mathcal{Z}^{\prime}, \quad i=0,1 .
\end{aligned}
$$

An example of a well-known family of reconstruction methods that can be approximated as linear is the penalized-likelihood family. These methods obtain the estimated image by maximizing an objective function

$$
\hat{\boldsymbol{f}}(\boldsymbol{y})=\arg \max _{\boldsymbol{f} \geq \mathbf{0}}[D(\boldsymbol{y}, \mathcal{A} \boldsymbol{f})-R(\boldsymbol{f})]
$$

where the objective function is composed of a data-fit term $D(\cdot, \cdot)$ and a regularization term $R(\cdot)$. The nonnegativity constraint $f \geq 0$ is used in emission tomography, where $f$ is a radioactivity distribution. A subset of penalized-likelihood methods to which we will refer later are penalized weighted least-squares (PWLS) methods. These make use of a data-fit term that is quadratic in the object $f$

$$
D(\boldsymbol{y}, \mathcal{A} \boldsymbol{f})=-(\boldsymbol{y}-\mathcal{A} \boldsymbol{f})^{\prime} \boldsymbol{W}(\boldsymbol{y}-\boldsymbol{A} \boldsymbol{f})
$$

for some measurement-domain weighting matrix $\boldsymbol{W}$. When the regularization term is also quadratic in $\boldsymbol{f}$, i.e.,

$$
R(f)=\boldsymbol{f}^{\prime} \mathcal{R} f
$$

for some linear operator $\mathcal{R}$, we have quadratically penalized WLS (QPWLS) reconstruction. 
In general, penalized-likelihood tomographic reconstruction can be approximated as linear, except maybe when enforcing the nonnegativity constraint [29]. Here, we assume that the signal of interest appears on a background that is sufficiently high to render the nonnegativity constraint inactive around the signal location, so these reconstructors can be considered linear near the signal. For example, if the nonnegativity constraint is ignored, one can show that QPWLS reconstruction with data-fit term (13) and regularization term (14) is equivalent to taking $\hat{\boldsymbol{f}}(\boldsymbol{y})=\left(\mathcal{A}^{\prime} \boldsymbol{W} \mathcal{A}+\mathcal{R}\right)^{-1} \mathcal{A}^{\prime} W \boldsymbol{y}$, which corresponds to the linear reconstructor

$$
\mathcal{Z}=\left(\mathcal{A}^{\prime} \boldsymbol{W} \mathcal{A}+\mathcal{R}\right)^{-1} \mathcal{A}^{\prime} \boldsymbol{W}
$$

For $\mathcal{R}=\mathbf{0}$, we get the unregularized WLS reconstructor, which simply maximizes the data-fit term (13).

\section{Observer Models}

The term observer refers to the entity making the decision between hypotheses $H_{0}$ and $H_{1}$. Observers acting directly on the measurement $\boldsymbol{y}$ would be very likely to grossly overestimate human observer performance, since humans have difficulty distinguishing small features in a sinogram. In the following, we will focus on observers that are applied to the reconstructed image $\hat{\boldsymbol{f}}$, since this situation better corresponds to common imaging practice.

We consider observers that decide between hypotheses $H_{0}$ and $H_{1}$ based on the reconstructed image $\hat{f}$ and a decision rule that, similarly to Neyman-Pearson tests, compares some scalar test statistic

$$
t=t(\hat{f})=t(\mathcal{Z} y)
$$

to some threshold T

$$
\text { Decide } H_{1} \text { if } t(\hat{\boldsymbol{f}})>T, \quad \text { otherwise decide } H_{0},
$$

where $t(\cdot)$ is the discriminant function and $T$ is independent of the data. The specific form of the discriminant function depends on the observer model considered. Our goal is to optimize the reconstructor $\mathcal{Z}$ with respect to the performance of various observer models of interest in the detection of $\boldsymbol{f}_{s}$.

\section{E. Figures of Merit}

One can quantify the detection performance of an observer by tracing its receiver operating characteristic (ROC) curve, a plot of the probability of a true positive (deciding that $H_{1}$ is true when $H_{1}$ is actually true) versus the probability of a false positive (deciding that $H_{1}$ is true when $H_{0}$ is actually true). The curve is traced by varying the decision threshold $T$ and the area under the curve (AUC) is a common figure of merit for observer performance. Another figure of merit is the signal-to-noise ratio (SNR), defined as

$$
\mathrm{SNR}=\frac{\mathrm{E}\left[t \mid H_{1}\right]-\mathrm{E}\left[t \mid H_{0}\right]}{\sqrt{\frac{1}{2} \operatorname{Var}\left\{t \mid H_{1}\right\}+\frac{1}{2} \operatorname{Var}\left\{t \mid H_{0}\right\}}} .
$$

In the case where the test statistic $t$ is Gaussian-distributed under both hypotheses, the SNR is especially useful, since it is monotonically related to the AUC [11, p. 819].

\section{OPtimal ReCONSTRUCTION FOR Signal DeteCtion}

\section{A. Ideal Observer}

The discriminant function of the ideal observer in a Bayesian sense is the well-known likelihood ratio [5, p. 26], or any monotonic function, such as the logarithm, of the likelihood ratio

$$
t_{\circ}(\hat{\boldsymbol{f}})=\log \left(p\left(\hat{\boldsymbol{f}}(\boldsymbol{y}) \mid H_{1}\right) / p\left(\hat{\boldsymbol{f}}(\boldsymbol{y}) \mid H_{0}\right)\right) .
$$

The ideal discriminant is generally nonlinear in $\hat{f}$. Even if the reconstructed image $\hat{f}$ is assumed to be Gaussian-distributed, the $\log$-likelihood ratio is quadratic in $\hat{f}$ and, thus, not Gaussian-distributed itself. Therefore, one would have to optimize the AUC of this test statistic directly rather than work with the SNR. However, the ideal observer is generally not used to evaluate reconstruction methods, since its performance is invariant to any nonsingular data transformation [30, Section 10.1]. The observer models commonly used in the literature to evaluate image reconstruction methods are linear.

\section{B. Generic Linear Observers}

Linear observer models facilitate analysis and they have been found to capture the suboptimality of human observers [31], [32]. Therefore, we focus hereafter on observers with a general linear discriminant of the form

$$
t(\boldsymbol{y})=\boldsymbol{w}^{\prime} \hat{\boldsymbol{f}}(\boldsymbol{y})
$$

for some template $\boldsymbol{w}$, which is a real vector in the same space as $\hat{f}$. Combining the linear discriminant in (18) with the reconstruction moments from (10) and (11) yields the following expressions for the moments of $t=t(\boldsymbol{y})$ :

$$
\begin{aligned}
\mathrm{E}\left[t \mid H_{1}\right]-\mathrm{E}\left[t \mid H_{0}\right] & =\boldsymbol{w}^{\prime} \mathcal{Z} \overline{\boldsymbol{y}} \\
\operatorname{Var}\left\{t \mid H_{i}\right\} & =\boldsymbol{w}^{\prime} \mathcal{Z} \Pi_{i} \mathcal{Z}^{\prime} \boldsymbol{w}, \quad i=0,1 .
\end{aligned}
$$

Hereafter, we assume that the test statistic $t$ is Gaussian-distributed and focus on maximizing the SNR, in which case the AUC is also maximized. For a linear observer $\boldsymbol{w}$ and a linear reconstructor $\mathcal{Z}$, the test statistic $t$ is a weighted sum of the elements of the measurement vector $\boldsymbol{y}$, so usually $t$ can be approximated as Gaussian-distributed by the central limit theorem. Furthermore, the probability distribution of an image $\hat{f}$ reconstructed from Poisson data $\boldsymbol{y}$ through a penalized-likelihood method of the form (12) can be approximated as Gaussian [33]. This is an additional argument for considering $t$ to be Gaussian-distributed when it is the product of a linear observer $w$ applied on an image $\hat{f}$ reconstructed by a penalized-likelihood algorithm.

Substituting the moment expressions (19) and (20) into (17) yields the SNR of the generic linear observer in (18)

$$
\mathrm{SNR}_{\text {lin }}^{2}=\frac{\left(\boldsymbol{w}^{\prime} \mathcal{Z} \bar{y}\right)^{2}}{\boldsymbol{w}^{\prime} \mathcal{Z} \overline{\boldsymbol{\Pi}} \mathcal{Z}^{\prime} \boldsymbol{w}}=\frac{\boldsymbol{w}^{\prime} \mathcal{Z}\left(\overline{\boldsymbol{y}} \overline{\boldsymbol{y}}^{\prime}\right) \mathcal{Z}^{\prime} \boldsymbol{w}}{\boldsymbol{w}^{\prime} \mathcal{Z} \check{\boldsymbol{\Pi}}^{\prime} \mathcal{Z}^{\prime} \boldsymbol{w}}
$$

where

$$
\check{\mathbf{\Pi}} \triangleq \frac{1}{2} \boldsymbol{\Pi}_{1}+\frac{1}{2} \boldsymbol{\Pi}_{0}
$$

is the unconditional covariance of the data. The left-hand side of the SNR expression (21) has the form of a generalized Rayleigh 
quotient. This form is maximized with respect to $\mathcal{Z}^{\prime} \boldsymbol{w}$ when (e.g., see [34, p. 120])

$$
\mathcal{Z}^{\prime} w \propto \check{\boldsymbol{\Pi}}^{-1} \overline{\boldsymbol{y}}
$$

When the observer template $\boldsymbol{w}$ and the reconstructor $\mathcal{Z}$ satisfy the condition (23), the observer at hand achieves the maximum SNR that is attainable with any combination of a linear observer and linear reconstruction method. Substituting the optimality condition (23) into the SNR expression (21) yields:

$$
\mathrm{SNR}_{\text {lin }}^{2} \leq \overline{\boldsymbol{y}}^{\prime} \check{\mathbf{\Pi}}^{-1} \overline{\boldsymbol{y}} \triangleq \mathrm{SNR}_{\operatorname{lin}_{o}}^{2} .
$$

The upper bound on the right-hand side of (24) is the maximum SNR attainable by any linear observer and linear reconstructor in the detection task at hand.

For emission tomography, substituting (7) and (8) into (22) yields

$$
\check{\Pi}=\Pi+\mathcal{A K}_{f} \mathcal{A}^{\prime}
$$

where $\boldsymbol{\Pi} \triangleq \operatorname{diag}\left\{\boldsymbol{\mathcal { A }}\left(\overline{\boldsymbol{f}}_{b}+(1 / 2) \overline{\boldsymbol{f}}_{s}\right)+\boldsymbol{r}\right\}$ is the imaging noise covariance for the unconditional mean object and $\mathcal{K}_{f} \triangleq \mathcal{K}_{b}+$ $(1 / 2) \mathcal{K}_{s}$ is the unconditional covariance of the object. Since $\Pi, \mathcal{K}_{b}$ and $\mathcal{K}_{s}$ are positive definite, so is $\check{\Pi}$ in (25), thus the ratio in (21) is well-defined provided $\mathcal{Z}^{\prime} \boldsymbol{w}$ is nonzero. Substituting (6) and (25) into (24) yields the optimal SNR for the detection task at hand in emission tomography

$$
\mathrm{SNR}_{\operatorname{lin}_{\circ}}^{2}=\overline{\boldsymbol{f}}_{s}^{\prime} \check{\mathcal{F}}_{s}
$$

where we define

$$
\begin{aligned}
\check{\mathcal{F}} & \triangleq \mathcal{A}^{\prime} \check{\Pi}^{-1} \mathcal{A}=\mathcal{A}^{\prime}\left(\boldsymbol{\Pi}+\mathcal{A} \mathcal{K}_{f} \mathcal{A}^{\prime}\right)^{-1} \mathcal{A} \\
& =\left(\mathcal{I}+\mathcal{F} \mathcal{K}_{f}\right)^{-1} \mathcal{F}=\mathcal{F}\left(\mathcal{I}+\mathcal{K}_{f} \mathcal{F}\right)^{-1}
\end{aligned}
$$

where $\mathcal{I}$ is the identity operator, and $\mathcal{F} \triangleq \mathcal{A}^{\prime} \Pi^{-1} \mathcal{A}$. From (27) we derive (28) using the "push-through" identity $\mathcal{A}(\mathcal{I}+$ $\mathcal{B A})^{-1}=(\mathcal{I}+\mathcal{A B})^{-1} \mathcal{A}$ [35]. The operators $\breve{\mathcal{F}}$ and $\mathcal{F}$ have a form analogous to that of the Fisher information matrix [5, p. 80] for estimation problems that involve a linear system and additive Gaussian noise. In the SKE/BKE case, we have $\breve{\mathcal{F}}=\mathcal{F}$.

A simple combination that satisfies the optimality condition (23) is $\mathcal{Z}=\mathcal{I}$ (which is not a reconstruction method) and $\boldsymbol{w}=\check{\boldsymbol{\Pi}}^{-1} \overline{\boldsymbol{y}}$, which corresponds to the Hotelling observer (see Section III-C1) for detection in the raw measurement domain rather than the reconstruction domain. However, even when we restrict attention to observers that are applied to reconstructed images, usually there are still many ways to satisfy (23), as the analysis that follows indicates.

\section{Fisher Observers and Reconstructors}

As shown in more detail later, several of the mathematical observers that have been proposed in the literature can achieve the optimal SNR when paired with simple reconstructors that correspond to some power of the Fisher information operator
$\check{\mathcal{F}}$ applied to a backprojection of the data. For lack of a better term we refer to this family of reconstruction methods as Fisher reconstructors and we allow them to include a regularization component

$$
\mathcal{Z}_{q} \triangleq \mathcal{H}^{(q)} \mathcal{A}^{\prime} \check{\Pi}^{-1}=(\check{\mathcal{F}}+\mathcal{R})^{(q)} \mathcal{A}^{\prime} \check{\boldsymbol{\Pi}}^{-1}
$$

for some $q \in \mathbb{R}$ and $\mathcal{H} \triangleq \check{\mathcal{F}}+\mathcal{R}$ for some regularization operator $\mathcal{R}$. We use the notation

$$
\mathcal{H}^{(q)} \triangleq \begin{cases}\mathcal{H}^{q}, & q \geq 0 \\ \left(\mathcal{H}^{\dagger}\right)^{-q}, & q<0\end{cases}
$$

where "†" denotes a pseudoinverse. ${ }^{1}$ Typically the regularizer $\mathcal{R}$ is chosen so that $\mathcal{H}$ is nonsingular. The reason we allow $\mathcal{H}$ to be singular in the analysis that follows is to accommodate the unregularized case $\mathcal{R}=\mathbf{0}$, since $\breve{\mathcal{F}}$ itself may be singular.

The estimated image produced by the Fisher reconstructor (29) is

$$
\hat{\boldsymbol{f}}=\mathcal{Z}_{q} \boldsymbol{y}=\mathcal{H}^{(q)} \mathcal{A}^{\prime} \check{\boldsymbol{\Pi}}^{-1} \boldsymbol{y}=\mathcal{H}^{(q)} \mathcal{A}^{\prime}\left(\boldsymbol{\Pi}+\mathcal{A K}_{f} \mathcal{A}^{\prime}\right)^{-1} \boldsymbol{y}
$$

which is a kind of weighted backprojection with a (perhaps somewhat unusual) postfilter. For $q<0$, this postfilter is something like a regularized deconvolver. For $q=-1$ in particular, the Fisher reconstructor yields

$$
\hat{f}=\mathcal{H}^{\dagger} \mathcal{A}^{\prime} \check{\boldsymbol{\Pi}}^{-1} \boldsymbol{y}
$$

so it is equivalent to the QPWLS estimator from (15) with $\boldsymbol{W}=\check{\boldsymbol{\Pi}}^{-1}$.

Interestingly, for any Fisher reconstructor, even an unregularized one, there is a corresponding linear observer that achieves optimal detection performance. We refer to this observer family as Fisher observers

$$
\boldsymbol{w}_{p} \triangleq \boldsymbol{\mathcal { H }}^{(p)} \overline{\boldsymbol{f}}_{s}
$$

for some $p \in \mathbb{R}$. For a reconstructor of the family (29) and an observer of the family (31) we have

$$
\mathcal{Z}_{q}^{\prime} \boldsymbol{w}_{p}=\check{\mathbf{\Pi}}^{-1} \mathcal{A} \mathcal{H}^{(q)} \mathcal{H}^{(p)} \overline{\boldsymbol{f}}_{s} .
$$

By comparing (32) with (23) for $\overline{\boldsymbol{y}}=\mathcal{A} \bar{f}_{s}$ we see that the choice $p=-q$ leads to the optimal SNR, i.e., the observer $\boldsymbol{w}_{-q}$ achieves optimal SNR when applied to images produced by the reconstructor $\mathcal{Z}_{q}$. This is true even for $\mathcal{R}=\mathbf{0}$ and singular $\breve{\mathcal{F}}$, as we can show by using the fact that $\mathcal{B}\left(\mathcal{B}^{\prime} \mathcal{B}\right)^{(q)}\left(\mathcal{B}^{\prime} \mathcal{B}\right)^{(-q)}=\mathcal{B}$ for any $\mathcal{B}$, which we obtain by singular value decomposition of $\mathcal{B}$. Substituting $\mathcal{B}=\check{\Pi}^{-1 / 2} \mathcal{A}$ yields the desired result. Fig. 1 shows template profiles for some of the observers in (31). The profile shape for $p=0.5$ especially is reminiscent of those estimated from human observers (e.g., see [19]).

\footnotetext{
${ }^{1}$ We follow [36, Definition 6.2.1 of the pseudoinverse]. An immediate result of this definition is that for nonsingular matrices the pseudoinverse is the same as the inverse [36, Theorem 6.2.13]. In this notation, we have $\mathcal{H}^{(-1)}=\boldsymbol{H}^{\dagger}=$ $\mathcal{H}^{-1}$ whenever $\boldsymbol{H}$ is nonsingular.
} 


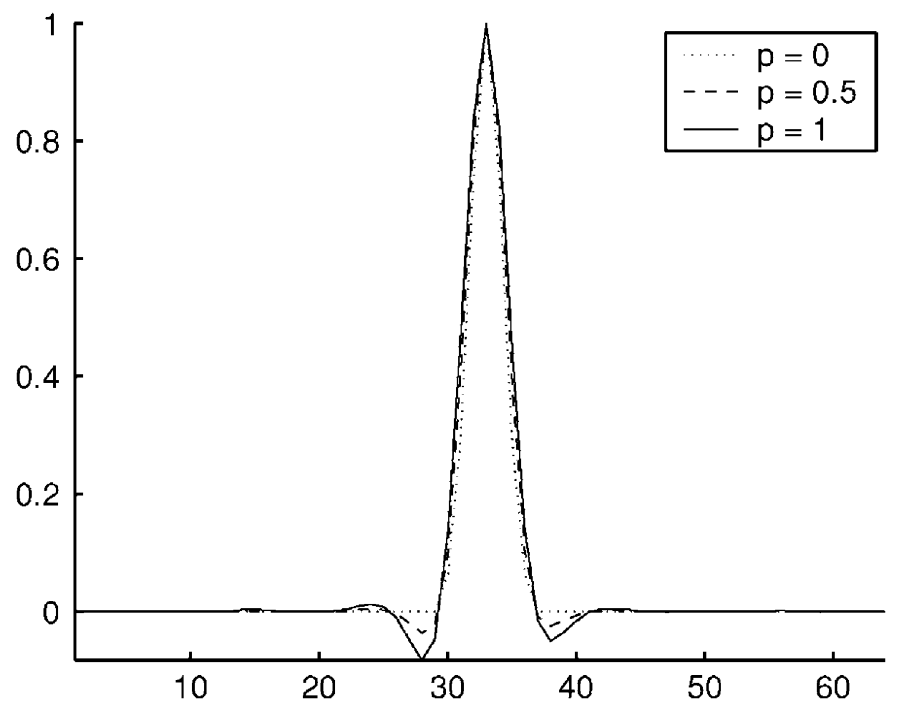

Fig. 1. Profiles through the center of (normalized) Fisher observer templates $\boldsymbol{w}_{p}$ for $p=0,0.5,1$. Note that $\boldsymbol{w}_{0}=\overline{\boldsymbol{f}}_{s}$.

Fisher reconstructors with $\mathcal{R}=\mathbf{0}$ appear to be largely devoid of regularization. ${ }^{2}$ However, even for these reconstructors, there are corresponding observers that can achieve optimal performance in the detection task considered here. As shown in the following sections, some well-known observers from the literature fall within this category. This means that, if the reconstructed images are meant to be interpreted by these observers, regularization is not essential in the reconstruction process, at least for the simple detection task at hand.

1) Hotelling Observer $(\mathrm{HO})$ : The $\mathrm{HO}$ utilizes the optimal linear discriminant in a maximum-SNR sense, which, similarly to (23), can be shown to be proportional to

$$
\boldsymbol{w}_{\mathrm{HO}} \triangleq \boldsymbol{K}_{\hat{\boldsymbol{f}}}^{\dagger}\left(\mathrm{E}\left[\hat{\boldsymbol{f}} \mid H_{1}\right]-\mathrm{E}\left[\hat{\boldsymbol{f}} \mid H_{0}\right]\right)
$$

where $\mathcal{K}_{\hat{\boldsymbol{f}}} \triangleq(1 / 2) \operatorname{Cov}\left\{\hat{\boldsymbol{f}} \mid H_{1}\right\}+(1 / 2) \operatorname{Cov}\left\{\hat{\boldsymbol{f}} \mid H_{0}\right\}$. Substituting the moments of $\hat{f}$ from (10) and (11) into (33) yields

$$
\boldsymbol{w}_{\mathrm{HO}}=\left(\mathcal{Z} \check{\boldsymbol{\Pi}}^{\prime}\right)^{\dagger} \mathcal{Z} \overline{\boldsymbol{y}}
$$

and, thus

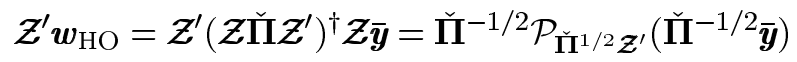

where $\mathcal{P}_{\check{\Pi}^{1 / 2}} \mathcal{Z}^{\prime}(\cdot)$ denotes the orthogonal projection of a vector onto $\mathcal{R}_{\check{\Pi}^{1 / 2} \mathcal{Z}^{\prime}}$, the range space of $\check{\Pi}^{1 / 2} \mathcal{Z}^{\prime}$. By comparing (35) with (23) we find that the HO achieves optimal SNR for any $\mathcal{Z}$ that satisfies $\check{\boldsymbol{\Pi}}^{-1 / 2} \overline{\boldsymbol{y}} \in \mathcal{R}_{\check{\Pi}^{1 / 2}} \mathcal{Z}^{\prime}$ or, equivalently

$$
\check{\Pi}^{-1} \overline{\boldsymbol{y}} \in \mathcal{R}_{\mathcal{z}^{\prime}}
$$

${ }^{2}$ For $\mathcal{R}=\mathbf{0}$ and $q>-1$, one could construe the Fisher reconstructor as being marginally regularized since it entails somewhat "less deconvolution" than the unregularized WLS reconstructor. However, this type of "regularization" does not improve the condition number in the case of singular $\breve{\mathcal{F}}$, and it is unlike most regularization methods described in the literature.
There are a multitude of choices of $\mathcal{Z}$ that satisfy the mild condition in (36). For example, any $\mathcal{Z}_{q}$ of the Fisher reconstructor family (29) satisfies (36), as long as $\overline{\boldsymbol{y}}$ is in the range space of $\mathcal{A}$ (e.g., $\overline{\boldsymbol{y}}=\mathcal{A} \overline{\boldsymbol{f}}_{s}$ ). This is true even for a $\mathcal{Z}_{q}$ with $\mathcal{R}=\mathbf{0}$ and singular $\breve{\mathcal{F}}$, following an argument similar to the previous section. Thus, the HO achieves the ideal SNR for any Fisher reconstructor, regularized or not. This result is consistent with the fact that linear transformations of the data do not affect the performance of the optimal linear observer [30, Section 10.2] (except when the transformation operator does not have a right inverse, in which case performance degrades). Note that for $\overline{\boldsymbol{y}}=\boldsymbol{\mathcal { A f }} \overline{\boldsymbol{f}}_{s}, \boldsymbol{\mathcal { R }}=\mathbf{0}$, and $q \neq 0$ the HO template in (34) becomes $\boldsymbol{w}_{\mathrm{HO}}=\breve{\mathcal{F}}^{(-q)} \overline{\boldsymbol{f}}_{s}$, which is the Fisher observer template in (31) with $p=-q$, and the optimality of $\mathcal{Z}_{q}$ for this observer follows the previous section.

2) Prewhitening $(P W)$ Observer: The template of the PW observer is given by

$$
\boldsymbol{w}_{\mathrm{PW}} \triangleq \operatorname{Cov}\left\{\hat{\boldsymbol{f}} \mid H_{0}\right\}^{\dagger}\left(\mathrm{E}\left[\hat{\boldsymbol{f}} \mid H_{1}\right]-\mathrm{E}\left[\hat{\boldsymbol{f}} \mid H_{0}\right]\right) \text {. }
$$

Substituting the moments of $\hat{\boldsymbol{f}}$ from (10) and (11) into (37) yields

$$
\boldsymbol{w}_{\mathrm{PW}}=\left(\mathcal{Z} \boldsymbol{\Pi}_{0} \mathcal{Z}^{\prime}\right)^{\dagger} \mathcal{Z} \overline{\boldsymbol{y}}
$$

and, thus

$$
\mathcal{Z}^{\prime} \boldsymbol{w}_{\mathrm{PW}}=\mathcal{Z}^{\prime}\left(\mathcal{Z} \boldsymbol{\Pi}_{0} \mathcal{Z}^{\prime}\right)^{\dagger} \mathcal{Z} \overline{\boldsymbol{y}}=\boldsymbol{\Pi}_{0}^{-1 / 2} \mathcal{P}_{\boldsymbol{\Pi}_{0}^{1 / 2} \mathcal{Z}^{\prime}}\left(\boldsymbol{\Pi}_{0}^{-1 / 2} \overline{\boldsymbol{y}}\right)
$$

By comparing (38) with (23) we find that the PW observer achieves optimal SNR for any $\mathcal{Z}$ that satisfies

$$
\mathcal{P}_{\boldsymbol{\Pi}_{0}^{1 / 2} \mathcal{z}^{\prime}}\left(\boldsymbol{\Pi}_{0}^{-1 / 2} \overline{\boldsymbol{y}}\right) \propto \boldsymbol{\Pi}_{0}^{1 / 2} \check{\boldsymbol{\Pi}}^{-1} \overline{\boldsymbol{y}}
$$

which in turn implies that $\boldsymbol{\Pi}_{0}^{-1 / 2} \overline{\boldsymbol{y}}-c \boldsymbol{\Pi}_{0}^{1 / 2} \check{\boldsymbol{\Pi}}^{-1} \overline{\boldsymbol{y}}$ must be orthogonal to $\mathcal{R}_{\boldsymbol{\Pi}_{0}^{1 / 2} \mathcal{Z}^{\prime}}$ for any constant $c$. This finally leads to the requirement that

$$
\mathcal{Z} \bar{y} \propto \mathcal{Z} \Pi_{0} \check{\Pi}^{-1} \bar{y}
$$

i.e., that the sinograms $\overline{\boldsymbol{y}}$ and $\boldsymbol{\Pi}_{0} \check{\boldsymbol{\Pi}}^{-1} \overline{\boldsymbol{y}}$ yield the same reconstructed image but for a scaling constant. Thus, in general there is no linear reconstructor $\mathcal{Z}$ that leads to optimal SNR for the PW observer.

An exception to this is the SKE case, where $\check{\boldsymbol{\Pi}}=\boldsymbol{\Pi}_{0}$ and the PW observer is the same as the HO, so it can achieve optimal SNR for infinitely many choices of $\mathcal{Z}$. The minimal dependence of the SNR on $\mathcal{Z}$ for the HO is consistent with the observation of Qi et al. that performance of the PW observer in the SKE task is independent of smoothing method in the MAP case [21].

3) Nonprewhitening $(N P W)$ Observer: The template of the NPW observer is given by

$$
\boldsymbol{w}_{\mathrm{NPW}} \triangleq \mathrm{E}\left[\hat{\boldsymbol{f}} \mid H_{1}\right]-\mathrm{E}\left[\hat{\boldsymbol{f}} \mid H_{0}\right]
$$

Substituting the moments of $\hat{\boldsymbol{f}}$ from (10) into (39) yields

$$
\boldsymbol{w}_{\mathrm{NPW}}=\mathcal{Z} \bar{y}
$$


TABLE I

Fisher RECONSTRUCTORS THAT ALLOW COMMON OBSERVER MODELS TO ACHIEVE OptIMAL SNR IN KNOWN-LOCATION DETECTION TASKS

\begin{tabular}{|c||c|c|r|l|}
\hline Observer & $q$ & $\mathcal{R}$ & Best estimator & Interpretation \\
\hline Hotelling & $\mathbb{R}$ & $\succeq \mathbf{0}$ & $(\dot{\mathcal{F}}+\mathcal{R})^{(q)} \mathcal{A}^{\prime} \check{\Pi}^{-1} \boldsymbol{y}$ & Any Fisher reconstructor $($ e.g., WLS $)$ \\
NPW & $-1 / 2$ & $\mathbf{0}$ & $\check{\mathcal{F}}^{(-1 / 2)} \mathcal{A}^{\prime} \check{\boldsymbol{\Pi}}^{-1} \boldsymbol{y}$ & Partly deconvolved backprojection \\
ROI & 0 & - & $\mathcal{A}^{\prime} \check{\boldsymbol{\Pi}}^{-1} \boldsymbol{y}$ & Backprojection \\
\hline
\end{tabular}

and, thus, by (23) the NPW observer achieves optimal SNR for any $\mathcal{Z}$ that satisfies

$$
\mathcal{Z}^{\prime} \mathcal{Z} \overline{\boldsymbol{y}} \propto \check{\boldsymbol{\Pi}}^{-1} \overline{\boldsymbol{y}}
$$

For a $\mathcal{Z}_{q}$ of the Fisher reconstructor family (29) and $\overline{\boldsymbol{y}}=$ $\mathcal{A} \overline{\boldsymbol{f}}_{s}$, we have $\mathcal{Z}^{\prime} \mathcal{Z} \overline{\boldsymbol{y}}=\check{\boldsymbol{\Pi}}^{-1} \mathcal{A} \mathcal{H}^{(2 q)} \breve{\mathcal{F}}_{\boldsymbol{s}}$, so the optimal SNR is achieved when $\mathcal{R}=0$ and $q=-1 / 2$. This corresponds to the somewhat unusual Fisher reconstructor

$$
\mathcal{Z}=\check{\mathcal{F}}^{(-1 / 2)} \mathcal{A}^{\prime} \check{\Pi}^{-1}
$$

Whether there are other solutions that satisfy (41) is an open problem. (There is also the choice $\mathcal{Z}=\breve{\Pi}^{-1 / 2}$, which is not a reconstruction method. It is equivalent to the HO for sinogrambased detection.) Note that for the reconstructor (42) the NPW template in (40) becomes $\boldsymbol{w}_{\mathrm{NPW}}=\breve{\mathcal{F}}^{1 / 2} \overline{\boldsymbol{f}}_{s}$, which is the Fisher observer template in (31) with $p=1 / 2$.

The reconstructor in (42) yields the estimated image

$$
\hat{\boldsymbol{f}}=\check{\mathcal{F}}^{(-1 / 2)} \mathcal{A}^{\prime} \check{\boldsymbol{\Pi}}^{-1} \boldsymbol{y}=\check{\mathcal{F}}^{1 / 2}\left(\check{\mathcal{F}}^{\dagger} \mathcal{A}^{\prime} \check{\boldsymbol{\Pi}}^{-1} \boldsymbol{y}\right)
$$

which is the unregularized WLS estimate, given in (30) for $\mathcal{R}=$ $\mathbf{0}$, followed by the unusual shift-variant postfilter $\check{\mathcal{F}}^{1 / 2}$. This estimator is impractical for two reasons. First, even if $\mathcal{A}$ happens to have full rank, $\breve{\mathcal{F}}$ is usually very ill-conditioned, so computing the WLS solution (30) will require a multitude of iterations for any practical iterative algorithm. Secondly, the shift-variant postfilter $\breve{\mathcal{F}}^{1 / 2}$ would be computationally intensive to implement for typical problem sizes.

4) Region-of-Interest (ROI) Observer: The template of the ROI observer is given by

$$
\boldsymbol{w}_{\mathrm{ROI}} \triangleq \mathrm{E}\left[\boldsymbol{f}_{s} \mid H_{1}\right]=\overline{\boldsymbol{f}}_{\boldsymbol{s}} .
$$

Then, by (23) the ROI observer achieves optimal SNR for any $\mathcal{Z}$ that satisfies

$$
\mathcal{Z}^{\prime} \bar{f}_{s} \propto \check{\boldsymbol{\Pi}}^{-1} \overline{\boldsymbol{y}}
$$

Since the ROI template corresponds to the Fisher observer in (31) with $p=0$, the optimal SNR for $\overline{\boldsymbol{y}}=\boldsymbol{\mathcal { A }} \overline{\boldsymbol{f}}_{s}$ is achieved by the Fisher reconstructor with $q=0$, i.e.,

$$
\mathcal{Z}=\mathcal{A}^{\prime} \check{\Pi}^{-1} \text {. }
$$

Curiously, in this case

$$
\hat{f}=\mathcal{A}^{\prime} \check{\boldsymbol{\Pi}}^{-1} \boldsymbol{y}
$$

This is a very blurry estimate of $\boldsymbol{f}$, being simply unfiltered backprojection. Yet for the ROI observer it is optimal, and no amount of deconvolution will improve the SNR for this detection task, which is an indication that the task is too simple. The optimality of this blurry estimate is consistent with the demonstration in Qi et al. of the ROI observer (for a penalized-likelihood reconstructor with $\mathcal{R}=\beta \mathcal{I}$ ) approaching the PW observer's performance as $\beta \rightarrow \infty$ [21].

Alternatively to (43), the ROI template may be defined as containing ones wherever $\overline{\boldsymbol{f}}_{s}$ is nonzero and zeroes everywhere else. In this case, the optimal $\mathcal{Z}$ would be the backprojector in (44) followed by a diagonal operator that weighs the reconstructed image by the shape of $\overline{\boldsymbol{f}}_{s}$, i.e., $\mathcal{Z}=\operatorname{diag}\left\{\overline{\boldsymbol{f}}_{s}\right\} \mathcal{A}^{\prime} \check{\boldsymbol{\Pi}}^{-1}$. This weighting does not improve the resolution of the backprojection, so for either definition of the ROI template the conclusion remains that optimal performance is achieved with a very blurry estimator.

5) Summary of Fisher Observers and Reconstructors: For three of the specific observer models considered above, at least one reconstructor of the Fisher family (29) was found to achieve the highest SNR possible for linear observers and linear reconstructors. Table I summarizes these findings. Thus, the linear reconstructors $\mathcal{Z}$ that yield optimal detection performance for the HO, NPW, and ROI models need not include any form of regularization. This is true even if the system operator $\mathcal{A}$ is a matrix with less than full column rank. We conclude on theoretical grounds that regularization is not absolutely essential for the HO, NPW, and ROI observer models in the task of detecting a statistically varying signal of known location on a statistically varying background.

The optimality of blurry backprojections with respect to detection performance for the HO, NPW, and ROI models implies that spatial resolution is not important for these known-location tasks. This is consistent with the findings of Wagner $e t$ $a l$., who attempted to optimize imaging systems with respect to performance in a SKE/BKE task, only to find that a pinhole of very large size would be optimal [37]. The authors later found that the presence of background variability decreased the optimal aperture size [38]. Our analysis shows that, for a given imaging system $\mathcal{A}$, there are reconstructors that degrade the spatial resolution of the system but lead to the optimal detection performance attainable with any combination of a linear observer, a linear reconstructor, and the specific system. As we have shown, this holds even in the presence of signal and background variability. The optimal reconstructors depend on the degree of signal and background variability through $\breve{\mathcal{F}}$ and $\check{\boldsymbol{\Pi}}$, which depend on the object covariance $\mathcal{K}_{f}$.

Furthermore, there is a strong dependence of the optimal reconstruction method on the specific observer model considered. This implies that there is no universally optimal reconstruction method, even for the simple detection task considered here. As 
we mentioned in Section I, the apparent premise of human-observer studies in the literature is that humans do not perform as well as the ideal observer. Therefore, the fact that there exist "simple" reconstruction methods that allow the HO, NPW, and ROI observer models to achieve the ideal linear-detection SNR (which is also the overall ideal SNR for SKE tasks) suggests that these observer models, the tasks, or both are somehow inappropriate. We proceed with analyzing the effect of regularization on the performance of channelized observer models, which are known to correlate well with human observers for known-location tasks.

\section{Channelized Linear Observers}

1) Overview: Channelized observer models include a set of frequency-selective channels in an attempt either to construct an efficient basis for the approximation of the ideal linear observer [39], or to model the frequency selectivity that is believed to characterize human visual perception [9]. Here, we are interested primarily in the latter type of channel, since suboptimal observers are the focus for the purpose of image reconstruction optimization. However, the analysis that follows applies to either channel flavor.

Conceptually, channelized observers first pass the reconstructed image $\hat{\boldsymbol{f}}$ through a set of $M$ bandpass filters. The new feature vector $\hat{\boldsymbol{c}} \in \mathbb{C}^{M}$ is formed from the values of the filter outputs at the known location of the target signal center and can include additive noise

$$
\hat{\boldsymbol{c}}(\boldsymbol{y})=\mathcal{C}^{\prime} \hat{\boldsymbol{f}}(\boldsymbol{y})+\varepsilon_{\mathrm{int}}
$$

where $\mathcal{C}=\left[\mathcal{C}_{1}, \ldots, \mathcal{C}_{M}\right]$ consists of $M$ operators. The $m$ th of these operators applies the impulse response of the $m$ th bandpass filter and samples the output at the center of the target signal. Typically this filtering step is not invertible and it greatly reduces the dimensionality of the detection problem (e.g., $M=$ 4 in [40]). The internal noise vector $\varepsilon_{\text {int }}$ models inherent uncertainty in the observer's decisions and is assumed to be zeromean Gaussian with covariance matrix $\boldsymbol{\Pi}_{\text {int }}$.

A generic channelized linear observer forms its test statistic $t_{\text {ch }}$ by applying a template $\boldsymbol{w} \in \mathbb{C}^{M}$ to the output of the filter bank

$$
t_{\mathrm{ch}}(\boldsymbol{y})=\boldsymbol{w}^{\prime} \hat{\boldsymbol{c}}(\boldsymbol{y})
$$

For a linear reconstruction method $\mathcal{Z}$, combining the reconstruction moments from (10) and (11) with the channelized linear discriminant in (45) yields the following expressions for the moments of $t_{\mathrm{ch}}$ :

$$
\begin{aligned}
\mathrm{E}\left[t_{\mathrm{ch}} \mid H_{1}\right]-\mathrm{E}\left[t_{\mathrm{ch}} \mid H_{0}\right] & =\boldsymbol{w}^{\prime} \mathcal{C}^{\prime} \mathcal{Z} \overline{\boldsymbol{y}} \\
\operatorname{Var}\left[t_{\mathrm{ch}} \mid H_{i}\right] & =\boldsymbol{w}^{\prime}\left(\mathcal{C}^{\prime} \mathcal{Z} \boldsymbol{\Pi}_{i} \mathcal{Z}^{\prime} \mathcal{C}+\boldsymbol{\Pi}_{\mathrm{int}}\right) \boldsymbol{w}
\end{aligned}
$$

$i=0,1$. Combining expressions (46) and (47) with (17) yields the SNR of the channelized observer

$$
\mathrm{SNR}_{\mathrm{ch}}^{2}=\frac{\left(\boldsymbol{w}^{\prime} \mathcal{C}^{\prime} \mathcal{Z} \bar{y}\right)^{2}}{\boldsymbol{w}^{\prime} \mathcal{C}^{\prime} \mathcal{Z} \check{\boldsymbol{\Pi}} \mathcal{Z}^{\prime} \mathcal{C} \boldsymbol{w}+\boldsymbol{w}^{\prime} \boldsymbol{\Pi}_{\mathrm{int}} \boldsymbol{w}}
$$

We would like to find a reconstructor $\mathcal{Z}$ that optimizes the SNR in (48). However, if $\boldsymbol{\Pi}_{\text {int }} \neq \mathbf{0}$ is independent of $\mathcal{Z}$, then a $\mathcal{Z}$ of infinitely large norm would be optimal. Thus, one would need some constraint on $\mathcal{Z}$ to optimize the SNR in its most general form (48), but it is unclear what constraints would be suitable.

A special case occurs if we assume that the covariance of the internal noise in the channels is proportional to the covariance of $\mathcal{C}^{\prime} \hat{f}$ due to imaging noise and object variability. Specifically, we assume

$$
\Pi_{\text {int }}=c_{\text {int }} \mathcal{C}^{\prime} \mathcal{Z}^{\prime} \check{\Pi} \mathcal{Z C}
$$

for some constant of proportionality $c_{\mathrm{int}}$, which includes the special case of no internal noise for $c_{\text {int }}=0$. The model (49) is similar to the one proposed in [16], except that (49) does not presume independence of the internal noise in individual channels. Under the assumption (49) the SNR of the channelized linear observer in (48) becomes

$$
\begin{aligned}
\mathrm{SNR}_{\mathrm{ch}}^{2} & =\frac{1}{1+c_{\mathrm{int}}} \frac{\left(\boldsymbol{w}^{\prime} \mathcal{C}^{\prime} \mathcal{Z} \overline{\boldsymbol{y}}\right)^{2}}{\boldsymbol{w}^{\prime} \mathcal{C}^{\prime} \mathcal{Z} \check{\mathbf{M}} \mathcal{Z}^{\prime} \mathcal{C} \boldsymbol{w}} \\
& \leq \frac{1}{1+c_{\mathrm{int}}} \overline{\boldsymbol{y}}^{\prime} \check{\boldsymbol{\Pi}}^{-1} \overline{\boldsymbol{y}}=\frac{\mathrm{SNR}_{\mathrm{lin}}^{2}}{1+c_{\mathrm{int}}}
\end{aligned}
$$

The SNR upper bound in (50) is achieved for any $\mathcal{Z}$ that satisfies

$$
\mathcal{Z}^{\prime} \mathcal{C w} \propto \check{\boldsymbol{\Pi}}^{-1} \bar{y}
$$

similarly to the nonchannelized version in (21) and (23).

2) Channelized Hotelling Observer ( $\mathrm{CHO})$ : As mentioned in Section I, the CHO has been shown to be particularly successful in predicting human observer performance. It applies the optimal linear discriminant with respect to the output $\hat{\boldsymbol{c}}$ of the $M$-channel filter bank. This corresponds to the template

$$
\begin{aligned}
\boldsymbol{w}_{\mathrm{CHO}} & \triangleq \boldsymbol{\Pi}_{\hat{\boldsymbol{c}}}^{\dagger}\left(\mathrm{E}\left[\hat{\boldsymbol{c}} \mid H_{1}\right]-\mathrm{E}\left[\hat{\boldsymbol{c}} \mid H_{0}\right]\right) \\
& =\left(\mathcal{C}^{\prime} \mathcal{Z} \check{\boldsymbol{\Pi}}^{\prime} \mathcal{Z}^{\prime} \mathcal{C}+\boldsymbol{\Pi}_{\text {int }}\right)^{\dagger} \mathcal{C}^{\prime} \mathcal{Z} \overline{\boldsymbol{y}}
\end{aligned}
$$

where $\boldsymbol{\Pi}_{\hat{\boldsymbol{c}}} \triangleq(1 / 2) \operatorname{Cov}\left\{\hat{\boldsymbol{c}} \mid H_{1}\right\}+(1 / 2) \operatorname{Cov}\left\{\hat{\boldsymbol{c}} \mid H_{0}\right\}$. (The filters involved in $\mathcal{C}$ typically correspond to distinct frequency bands. If this is the case, then the covariance $\boldsymbol{\Pi}_{\hat{\boldsymbol{c}}}$ can be assumed to be nonsingular even when $\boldsymbol{\Pi}_{\text {int }}=\mathbf{0}$, so its pseudoinverse is the same as its inverse. Nevertheless, we use a pseudoinverse in the interest of generality.) By substituting the $\mathrm{CHO}$ template from (52) into the SNR expression (48), we find this observer's SNR

$$
\mathrm{SNR}_{\mathrm{CHO}}^{2}=\overline{\boldsymbol{y}}^{\prime} \mathcal{Z}^{\prime} \mathcal{C}\left(\mathcal{C}^{\prime} \mathcal{Z} \check{\boldsymbol{\Pi}} \mathcal{Z}^{\prime} \mathcal{C}+\boldsymbol{\Pi}_{\mathrm{int}}\right)^{\dagger} \mathcal{C}^{\prime} \mathcal{Z} \overline{\boldsymbol{y}}
$$

In the special case of the internal noise model (49), we have

$$
\begin{aligned}
\mathcal{Z}^{\prime} \mathcal{C} \boldsymbol{w}_{\mathrm{CHO}} & \propto \mathcal{Z}^{\prime} \mathcal{C}\left(\mathcal{C}^{\prime} \mathcal{Z} \check{\boldsymbol{\Pi}}^{\prime} \mathcal{C}\right)^{\dagger} \mathcal{C}^{\prime} \mathcal{Z} \overline{\boldsymbol{y}} \\
& =\check{\boldsymbol{\Pi}}^{-1 / 2} \mathcal{P}_{\check{\boldsymbol{\Pi}}^{1 / 2} \mathcal{Z}^{\prime} \mathcal{C}}\left(\check{\boldsymbol{\Pi}}^{-1 / 2} \overline{\boldsymbol{y}}\right)
\end{aligned}
$$

By comparing (54) to the optimality condition (51), we find that the SNR upper bound in (50) is achieved when $\mathcal{Z}$ satisfies

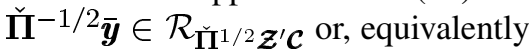

$$
\check{\Pi}^{-1} \bar{y} \in \mathcal{R}_{\mathcal{Z}^{\prime} \mathcal{C}}
$$


which is similar to (36). To find a $\mathcal{Z}$ that satisfies (55), consider a reconstructor of the form

$$
\mathcal{Z}=\mathcal{W C}\left(\mathcal{C}^{\prime} \mathcal{W C}\right)^{-1} \mathcal{G}^{\prime} \mathcal{A}^{\prime} \check{\Pi}^{-1}
$$

where $\mathcal{W}$ is any image-domain weighting operator and $\mathcal{G}$ is a mapping from $\mathbb{C}^{M}$ to object space. For the $\mathcal{Z}$ in (56), we have $\mathcal{Z}^{\prime} \mathcal{C}=\check{\Pi}^{-1} \mathcal{A G}$, so the optimality condition (55) is satisfied if

$$
\overline{\boldsymbol{y}} \in \mathcal{R}_{\mathcal{A G}} .
$$

As long as $\mathcal{G}$ can be chosen to satisfy (57), the unregularized reconstructor $\mathcal{Z}$ given in (56) allows the $\mathrm{CHO}$ to achieve the optimal SNR. For emission tomography, where $\overline{\boldsymbol{y}}=\mathcal{A} \overline{\boldsymbol{f}}_{s}$, there is always a way to choose $\mathcal{G}$ to satisfy (57); choosing $\mathcal{G}$ so that one of its "columns" is proportional to $\bar{f}_{s}$ is the most obvious way.

The rather unconventional family of reconstructors in (56), although ensuring optimal SNR for the CHO under the internal noise model (49), does not produce what we usually consider to be reconstructed images. In essence, the HO template for detection in the raw measurement domain $\left(\boldsymbol{w}=\check{\boldsymbol{\Pi}}^{-1} \overline{\boldsymbol{y}}\right)$ is hidden in one of the rows of such a reconstructor. Thus, it is of interest to know whether reconstructors that are more conventional than (56) can be combined with the $\mathrm{CHO}$ to achieve SNR values close to the optimal and, if so, whether these reconstructors must include regularization. As shown below, such insight can be gained by using approximations to the SNR expression in (53) that are based on local Fourier-domain analysis. Such approximations also facilitate analysis of internal noise models other than (59). In the following, we first provide a brief review of local Fourier-domain approximations and we then use them to analyze the SNR of the $\mathrm{CHO}$ for one unregularized and one regularized reconstructor example.

3) Local Fourier Analysis of CHO Performance: For the purposes of the Fourier analysis that follows, we will assume to have a discrete representation of the object ${ }^{3} \boldsymbol{f} \in \mathbb{R}^{n_{\mathrm{p}}}$. Let $\mathcal{U}$ be a discrete Fourier operator, mapping an object in $\mathbb{R}^{n_{\mathrm{p}}}$ to some vector in $\mathbb{C}^{n_{\mathrm{p}}}$ known as the spectrum of the object. The Fourier operator $\mathcal{U}$ is linear and, due to the shift property of the Fourier transform, it can be defined through its action on an object $\boldsymbol{e}_{0}$ that consists of an impulse centered at the origin

$$
\mathcal{U} \boldsymbol{e}_{0}=\frac{1}{\sqrt{n_{\mathrm{p}}}} \mathbf{1}
$$

where 1 is the vector of $n_{\mathrm{p}}$ ones. Without loss of generality, we choose the origin of the Fourier transform to coincide with the location where the signal $\bar{f}_{s}$ is centered. (The $\left(1 / \sqrt{n_{\mathrm{p}}}\right)$ factor results simply from using the orthonormal form of the DFT.)

Let $\lambda \in \mathbb{C}^{n_{\mathrm{p}}}$ be the local frequency response of the Fisher information operator $\mathcal{F}$ at the origin, defined by

$$
\lambda \triangleq \sqrt{n_{\mathrm{p}}} \boldsymbol{U} \mathcal{F} \boldsymbol{e}_{0} .
$$

In general, the operator $\mathcal{F}$ is shift-variant, so its behavior when applied to an object other than the impulse $\boldsymbol{e}_{0}$ cannot be fully defined through $\lambda$. However, if the operator $\mathcal{F}$ is applied to an object that is contained within a small spatial extent around the

\footnotetext{
${ }^{3}$ Nevertheless, it is possible to generalize this analysis for an infinite-dimen-
} sional object space. origin, we can approximate the behavior of $\mathcal{F}$ using $\boldsymbol{\lambda}$, i.e., we can approximate $\mathcal{F}$ as shift-invariant within the spatial extent of the object. The closer the object comes to resembling an impulse, the greater the accuracy of this approximation. Thus, we approximate the operator $\mathcal{F}$ within a small spatial extent around the origin as

$$
\mathcal{F} \approx \mathcal{U}^{-1} \Lambda \mathcal{U}
$$

where $\boldsymbol{\Lambda} \triangleq \operatorname{diag}\{\lambda\}$. The elements of $\boldsymbol{\lambda}, \lambda_{k}, k=1, \ldots, n_{\mathrm{p}}$, contain the local frequency response of $\mathcal{F}$. Since $\mathcal{F}$ is symmetric nonnegative definite, we force the $\lambda_{k}$ 's to be real and nonnegative by discarding imaginary parts and setting negatives to zero. Locally shift-invariant approximations of $\mathcal{F}$ have proven to be useful and accurate in several cases [20], [21], [33], [41]-[45].

We also use a locally shift-invariant approximation of the object covariance

$$
\mathcal{K}_{f} \approx \mathcal{U}^{-1} N \mathcal{U}
$$

where $N \triangleq \operatorname{diag}\left\{\nu_{k}, k=1, \ldots, n_{\mathrm{p}}\right\}$. The $\nu_{k}$ 's contain the local frequency response of $\mathcal{K}_{f}$ (i.e., the local power spectrum of the object $\boldsymbol{f}$ ) around the location of $\overline{\boldsymbol{f}}_{s}$. Although it may be reasonable to approximate the background $\boldsymbol{f}_{b}$ as locally stationary within the spatial extent of the signal, approximating the signal $\boldsymbol{f}_{s}$ itself as locally stationary would be less reasonable. That is, the approximation in (60) is much more accurate when the signal $f_{s}$ is deterministic or when the signal covariance $\mathcal{K}_{s}$ is negligible in comparison to the background covariance $\mathcal{K}_{b}$.

Using the approximations (59) and (60), we can start from (28) to derive the following locally shift-invariant approximation for the Fisher information operator $\breve{\mathcal{F}}$

$$
\begin{aligned}
\check{\mathcal{F}} & =\mathcal{F}\left(\mathcal{I}+\mathcal{K}_{f} \mathcal{F}\right)^{-1} \\
& \approx \mathcal{U}^{-1} \boldsymbol{\Lambda}(\boldsymbol{I}+\boldsymbol{N} \boldsymbol{\Lambda})^{-1} \mathcal{U}=\mathcal{U}^{-1} \check{\Lambda} \mathcal{U}
\end{aligned}
$$

where $\check{\Lambda} \triangleq \operatorname{diag}\left\{\check{\lambda}_{k}, k=1, \ldots, n_{\mathrm{p}}\right\}$ and $\check{\lambda}_{k} \triangleq\left(\lambda_{k}\right) /\left(1+\nu_{k} \lambda_{k}\right)$, which reduces to $\check{\Lambda}=\boldsymbol{\Lambda}$ in the SKE/BKE case.

As in [22]-[24], we also use the fact that $\mathcal{C}$ is a collection of filters to get its frequency-domain representation. Let $\boldsymbol{t}^{m} \in \mathbb{C}^{n_{\mathrm{p}}}$ denote the frequency response of the $m$ th bandpass filter. Then, the $m$ th operator in $\mathcal{C}$ has the form $\mathcal{C}_{m}=\mathcal{U}^{-1} \operatorname{diag}\left\{\boldsymbol{t}^{m}\right\} \mathcal{U} \boldsymbol{e}_{0}$ and, using (58), we get

$$
\mathcal{C}=\mathcal{U}^{-1} \boldsymbol{T}, \quad \boldsymbol{T}=\frac{1}{\sqrt{n_{\mathrm{p}}}}\left[\begin{array}{lll}
\boldsymbol{t}^{1} & \ldots & \boldsymbol{t}^{M}
\end{array}\right] .
$$

4) $\mathrm{CHO}$ and Fisher Reconstructors: For an unregularized Fisher reconstructor $\mathcal{Z}_{q}$, given by (29) with $\mathcal{R}=\mathbf{0}$, we can use the Fourier decompositions (61) and (62) along with (6) to obtain the approximations

$$
\begin{aligned}
\mathcal{C}^{\prime} \mathcal{Z}_{q} \overline{\boldsymbol{y}} & \approx \boldsymbol{T}^{\prime} \check{\boldsymbol{\Lambda}}^{(1+q)} \boldsymbol{X} \\
\mathcal{C}^{\prime} \mathcal{Z}_{q} \check{\boldsymbol{\Pi}} \mathcal{Z}_{q}^{\prime} \mathcal{C} & \approx \boldsymbol{T}^{\prime} \check{\boldsymbol{\Lambda}}^{(1+2 q)} \boldsymbol{T}
\end{aligned}
$$

where $X=\mathcal{U} \bar{f}_{s}$ is the spectrum of the mean target signal. The approximation (63) assumes that $\bar{f}_{s}$ is highly localized in space. The accuracy of the approximation (64) depends on how localized in space the channel responses are. However, when $\check{\Lambda}$ is positive definite, the expression (64) is exact for $q=-1 / 2$, 
so it may also be reasonable for $q$ near $-1 / 2$ (including $q=-1$ and $q=0$, which are the two cases of greatest practical interest). Approximation error plots for some values of $q$ were presented in [22].

Substituting the approximations (64) and (63) into (53) yields the following approximation for the SNR of the $\mathrm{CHO}$ when combined with an unregularized Fisher reconstructor

$\mathrm{SNR}_{\mathrm{CHO}, \mathrm{F}}^{2} \approx \boldsymbol{X}^{\prime} \check{\Lambda}^{(1+q)} \boldsymbol{T}\left(\boldsymbol{T}^{\prime} \check{\boldsymbol{\Lambda}}^{(1+2 q)} \boldsymbol{T}+\boldsymbol{\Pi}_{\mathrm{int}}\right)^{\dagger} \boldsymbol{T}^{\prime} \check{\boldsymbol{\Lambda}}^{(1+q)} \boldsymbol{X}$.

5) Channels With Disjoint Passbands: The CHO filters are sometimes assumed to be bandpass filters with disjoint passbands. In that case, the vectors $\boldsymbol{t}^{m}$ have disjoint nonzero entries and the $M \times M$ matrix on the right-hand side of (64) is diagonal, so we have

$$
\begin{aligned}
{\left[\boldsymbol{T}^{\prime} \check{\boldsymbol{\Lambda}}^{(1+q)} \boldsymbol{X}\right]_{m} } & =\frac{1}{\sqrt{n_{\mathrm{p}}}} \sum_{k \in \mathcal{T}_{m}} \phi_{k} \\
{\left[\boldsymbol{T}^{\prime} \check{\boldsymbol{\Lambda}}^{(1+2 q)} \boldsymbol{T}\right]_{m m} } & =\frac{1}{n_{\mathrm{p}}} \sum_{k \in \mathcal{T}_{m}} \psi_{k}
\end{aligned}
$$

where

$$
\phi_{k} \triangleq\left(t_{k}^{m}\right)^{*} X_{k} \check{\lambda}_{k}^{(1+q)}, \quad \psi_{k} \triangleq\left|t_{k}^{m}\right|^{2} \check{\lambda}_{k}^{(1+2 q)}
$$

$\mathcal{T}_{m}=\left\{k: t_{k}^{m} \neq 0\right\}$ is the passband of the $m$ th filter and “*” denotes complex conjugate.

If we furthermore combine the assumption of disjoint passbands with the internal noise model (49), the covariance $\boldsymbol{\Pi}_{\text {int }}$ becomes diagonal (to within approximation (64)) and the model (49) coincides with the one proposed in [16]. Under these assumptions the SNR approximation in (65) simplifies to

$$
\mathrm{SNR}_{\mathrm{CHO}, F}^{2} \approx \mathrm{SNR}_{1}^{2} \triangleq \frac{1}{1+c_{\mathrm{int}}} \sum_{m=1}^{M} \frac{\left|\sum_{k \in \mathcal{T}_{m}} \phi_{k}\right|^{2}}{\sum_{k \in \mathcal{T}_{m}} \psi_{k}} .
$$

We now examine the conditions under which the approximate SNR in (66) can reach the SNR upper bound in (50).

6) Achievability of the Optimal SNR: To determine how closely the approximate SNR in (66) can approach the SNR upper bound, define vectors $\boldsymbol{u}^{m}$ and $\boldsymbol{v}^{m}$ with elements

$$
\begin{aligned}
& u_{k}^{m} \triangleq X_{k} \check{\lambda}_{k}^{1 / 2} 1_{\left\{k \in \mathcal{T}_{m}\right\}}, \\
& v_{k}^{m} \triangleq t_{k}^{m} \check{\lambda}_{k}^{(q+1 / 2)}
\end{aligned}
$$

respectively. Then, rewriting (66) and using Cauchy's inequality yields

$$
\begin{aligned}
\mathrm{SNR}_{1}^{2} & =\frac{1}{1+c_{\text {int }}} \sum_{m=1}^{M} \frac{\left|\left\langle\boldsymbol{u}^{m}, \boldsymbol{v}^{m}\right\rangle\right|^{2}}{\left\|\boldsymbol{v}^{m}\right\|^{2}} \\
& \leq \frac{1}{1+c_{\text {int }}} \sum_{m=1}^{M}\left\|\boldsymbol{u}^{m}\right\|^{2} \\
& =\frac{1}{1+c_{\text {int }}} \sum_{k \in \mathcal{T}}\left|X_{k}\right|^{2} \check{\lambda}_{k} \\
& \leq \frac{1}{1+c_{\text {int }}} \sum_{k}\left|X_{k}\right|^{2} \check{\lambda}_{k} \\
& \approx \frac{1}{1+c_{\text {int }}} \overline{\boldsymbol{f}}_{s}^{\prime} \check{\mathcal{F}} \overline{\boldsymbol{f}}_{s}=\frac{\mathrm{SNR}_{\text {lin }}^{2}}{1+c_{\mathrm{int}}}
\end{aligned}
$$

where $\mathcal{T}=\cup_{m=1}^{M} \mathcal{T}_{m}$ denotes the combined passband of all $M$ channels. If the combined passband $\mathcal{T}$ does not contain all of the signal energy, then the SNR will be strictly less than the optimal SNR. This suboptimality is expected due to the dimensionality decrease caused by the channels.

Is there an unregularized Fisher reconstructor $\mathcal{Z}_{q}$ for which the upper bound in (67) can be achieved? Suppose that each channel filter has a flat passband, i.e., $t_{k}^{m}=1_{\left\{k \in \mathcal{T}_{m}\right\}}$. Then, there are two obvious cases where the SNR upper bound in (67) is achieved, as can be verified by substitution or by using the requirement $\boldsymbol{u}^{m} \propto \boldsymbol{v}^{m} \forall m$.

- If the $X_{k}$ 's are constant over each passband, then $q=0$ (the unfiltered backprojector) will be optimal.

- If the $\breve{\lambda}_{k}$ 's are also constant over each passband, then any $q \in \mathbb{R}$ will be optimal.

In practice, it may be unlikely that either the $\check{\lambda}_{k}$ 's or the $X_{k}$ 's are exactly uniform over each channel's passband, but if the passbands are reasonably narrow, then it is likely that these spectra will be approximately uniform over each passband. So to within the accuracy of the approximations considered above, one or more of these unregularized reconstructors will nearly achieve the highest SNR obtainable for the given CHO channels and internal noise model. Once again, regularization does not seem to play a crucial role, even for the $\mathrm{CHO}$.

7) CHO and QPWLS Reconstructors: The analysis in the previous section showed some situations in which one or more unregularized reconstructors allow the $\mathrm{CHO}$ to achieve (approximately) optimal SNR in the detection task at hand. We next examine the QPWLS family of regularized reconstructors, described in Section II-C, to explore how closely the CHO can approach the optimal SNR with a practical regularized reconstruction method and to examine the optimal choice of regularizer.

The general form of a QPWLS reconstructor is given in (15) and the commonly used weighting matrix $\boldsymbol{W}$ is the one corresponding to MAP estimation, i.e., $\boldsymbol{W}=(\operatorname{Cov}\{\boldsymbol{y} \mid \boldsymbol{f}=\overline{\boldsymbol{f}}\})^{-1}=$ $\Pi^{-1}$, where $\bar{f}$ is the unconditional mean of the object. For this $W$, the reconstructor in (15) becomes

$$
\mathcal{Z}=(\mathcal{F}+\mathcal{R})^{-1} \mathcal{A}^{\prime} \Pi^{-1}
$$

Note that the QPWLS reconstructor in (68) is the same as the Fisher reconstructor (29) with $q=-1$ only if $\mathcal{K}_{f}=\mathbf{0}$, i.e., only if there is no object variability. We assume throughout that the regularization operator $\mathcal{R}$ is chosen such that $\mathcal{F}+\mathcal{R}$ is positive definite. We would like to design $\mathcal{R}$ to optimize $\mathrm{CHO}$ detection performance in the QPWLS-reconstructed images.

To analyze CHO performance with QPWLS reconstruction, we assume that both $\mathcal{F}$ and $\mathcal{R}$ are diagonalized locally by a common operator (the Fourier operator $\mathcal{U}$ ). Specifically, we use (59), (60), (62) and also assume that

$$
\mathcal{R} \approx \mathcal{U}^{-1} \Omega \mathcal{U}
$$

where $\boldsymbol{\Omega} \triangleq \operatorname{diag}\left\{\omega_{k}, k=1, \ldots, n_{\mathrm{p}}\right\}$. The real, nonnegative $\omega_{k}$ 's contain the local frequency response of the regularizer around the location of $\overline{\boldsymbol{f}}_{s}$. Approximations of simultaneous diagonalization of $\mathcal{F}$ and $\mathcal{R}$ have been used by other investigators to analyze observer performance with penalized-likelihood reconstruction [20], [23], [24]. 
Substituting the QPWLS reconstructor (68) and the emission tomography measurement moments (6) and (25) into the SNR of the CHO from (53) yields

$$
\mathrm{SNR}_{\mathrm{CHO}, \mathrm{QPWLS}}^{2}=\overline{\boldsymbol{c}}^{\prime} \boldsymbol{\Pi}_{\hat{\boldsymbol{c}}}^{\dagger} \overline{\boldsymbol{c}}
$$

where

$$
\begin{aligned}
\overline{\boldsymbol{c}} & \triangleq \mathcal{C}^{\prime} \mathcal{H}^{-1} \mathcal{F} \bar{f}_{s} \\
\Pi_{\hat{c}} & \triangleq \mathcal{C}^{\prime} \mathcal{H}^{-1}\left(\mathcal{F}+\mathcal{F} \mathcal{K}_{f} \mathcal{F}\right) \mathcal{H}^{-1} \mathcal{C}+\Pi_{\mathrm{int}}
\end{aligned}
$$

and $\mathcal{H} \triangleq \mathcal{F}+\mathcal{R}$. Similarly to Section III-D4, we use the local Fourier approximations (59), (60), and (69) to obtain

$$
\begin{aligned}
\overline{\boldsymbol{c}} & \approx \boldsymbol{T}^{\prime} \boldsymbol{H}^{-1} \boldsymbol{\Lambda} \boldsymbol{X} \\
\boldsymbol{\Pi}_{\hat{c}} & \approx \boldsymbol{T}^{\prime} \boldsymbol{H}^{-1}\left(\boldsymbol{\Lambda}+\boldsymbol{\Lambda}^{2} \boldsymbol{N}\right) \boldsymbol{H}^{-1} \boldsymbol{T}+\boldsymbol{\Pi}_{\mathrm{int}}
\end{aligned}
$$

where $\boldsymbol{H} \triangleq \boldsymbol{\Lambda}+\boldsymbol{\Omega}=\operatorname{diag}\left\{\lambda_{k}+\omega_{k}, k=1, \ldots, n_{\mathrm{p}}\right\}$. Substituting (71) and (72) into (70) yields the following approximation for the SNR of the CHO when combined with a QPWLS reconstructor:

$$
\begin{aligned}
\mathrm{SNR}_{\mathrm{CHO}, \mathrm{QPWLS}}^{2} \approx & \boldsymbol{X}^{\prime} \boldsymbol{\Lambda} \boldsymbol{H}^{-1} \boldsymbol{T} \\
& \cdot\left(\boldsymbol{T}^{\prime} \boldsymbol{H}^{-1}\left(\boldsymbol{\Lambda}+\boldsymbol{\Lambda}^{2} \boldsymbol{N}\right) \boldsymbol{H}^{-1} \boldsymbol{T}+\boldsymbol{\Pi}_{\mathrm{int}}\right)^{\dagger} \\
& \cdot \boldsymbol{T}^{\prime} \boldsymbol{H}^{-1} \boldsymbol{\Lambda} \boldsymbol{X}
\end{aligned}
$$

8) Channels With Disjoint Passbands: Similarly to Section III-D5, when the CHO channels have disjoint frequency responses, the $M \times M$ matrix on the right-hand side of (72) becomes diagonal. We assume that its diagonal elements are nonzero, i.e., the system has some nonzero $\lambda_{k}$ for each passband. (If not, the noninformative passband could be eliminated.)

As in Section III-D5, we combine the assumption of disjoint passbands with the internal noise model (49). Then, the approximate SNR expression in (73) becomes

$$
\mathrm{SNR}_{\mathrm{CHO}, \mathrm{QPWLS}}^{2} \approx \mathrm{SNR}_{2}^{2} \triangleq \frac{1}{1+c_{\mathrm{int}}} \sum_{m=1}^{M} \frac{\left|\sum_{k \in \mathcal{T}_{m}} \phi_{k}\right|^{2}}{\sum_{k \in \mathcal{T}_{m}} \psi_{k}}
$$

where

$$
\phi_{k} \triangleq X_{k}\left(t_{k}^{m}\right)^{*} \frac{\lambda_{k}}{\lambda_{k}+\omega_{k}}, \quad \psi_{k} \triangleq\left|t_{k}^{m}\right|^{2} \frac{\lambda_{k}^{2}}{\check{\lambda}_{k}\left(\lambda_{k}+\omega_{k}\right)^{2}} .
$$

We now examine the conditions under which the approximate SNR in (74) can reach the SNR upper bound in (50).

9) Achievability of the Optimal SNR: To determine how closely the approximate SNR in (74) can approach the SNR upper bound, define vectors $\boldsymbol{u}^{m}$ and $\boldsymbol{v}^{m}$ with elements

$$
\begin{aligned}
& u_{k}^{m} \triangleq X_{k} \check{\lambda}_{k}^{1 / 2} 1_{\left\{k \in \mathcal{T}_{m}\right\}}, \\
& v_{k}^{m} \triangleq t_{k}^{m} \lambda_{k} / \check{\lambda}_{k}^{1 / 2}\left(\lambda_{k}+\omega_{k}\right)
\end{aligned}
$$

respectively. Then, rewriting (74) and using Cauchy's inequality yields

$$
\begin{aligned}
\mathrm{SNR}_{2}^{2} & =\frac{1}{1+c_{\text {int }}} \sum_{m=1}^{M} \frac{\left|\left\langle\boldsymbol{u}^{m}, \boldsymbol{v}^{m}\right\rangle\right|^{2}}{\left\|\boldsymbol{v}^{m}\right\|^{2}} \\
& \leq \frac{1}{1+c_{\text {int }}} \sum_{m=1}^{M}\left\|\boldsymbol{u}^{m}\right\|^{2} \\
& =\frac{1}{1+c_{\text {int }}} \sum_{k \in \mathcal{T}}\left|X_{k}\right|^{2} \check{\lambda}_{k} \\
& \leq \frac{1}{1+c_{\text {int }}} \sum_{k}\left|X_{k}\right|^{2} \check{\lambda}_{k} \\
& \approx \frac{1}{1+c_{\text {int }}} \overline{\boldsymbol{f}}_{s}^{\prime} \check{\mathcal{F}} \overline{\boldsymbol{f}}_{s}=\frac{\mathrm{SNR}_{\text {lino }}^{2}}{1+c_{\text {int }}} .
\end{aligned}
$$

Once again, if the combined passband $\mathcal{T}=\cup_{m=1}^{M} \mathcal{T}_{m}$ does not contain all of the signal energy, then the SNR will be strictly less than the optimal SNR.

The intermediate inequality in (75) becomes an equality if $\boldsymbol{u}^{m} \propto \boldsymbol{v}^{m} \forall m$. Suppose that each channel filter is an ideal bandpass filter over some frequency band, i.e., $t_{k}^{m}=1_{\left\{k \in \mathcal{T}_{m}\right\}}$. Then, for $\lambda_{k} \neq 0, X_{k} \neq 0$, the intermediate upper bound in (75) is achieved for example when

$$
\omega_{k}=\alpha \frac{\lambda_{k}}{X_{k} \check{\lambda}_{k}}-\lambda_{k}
$$

where the constant $\alpha \neq 0$ can be chosen arbitrarily. Using $\alpha \triangleq$ $2 \max _{k} X_{k} \check{\lambda}_{k}$ would keep the $\omega_{k}$ 's positive. To within approximations (59), (60), and (69), the local frequency response in (76) corresponds to the following nonnegative definite regularizer

$$
\mathcal{R}=\alpha\left(\mathcal{I}+\mathcal{F} \mathcal{K}_{f}\right)\left(\mathcal{U}^{-1} \operatorname{diag}\{\boldsymbol{X}\}^{-1} \mathcal{U}\right)-\mathcal{F}
$$

The $\mathcal{R}$ in (77) usually has a high-pass characteristic, so it could be construed as a regularization operator, but it is quite different from standard forms of regularization studied in the literature. Furthermore, substituting the $\mathcal{R}$ from (77) into the QPWLS reconstructor in (68) yields the estimator

$$
\hat{\boldsymbol{f}}=\frac{1}{\alpha}\left(\mathcal{U}^{-1} \operatorname{diag}\{\boldsymbol{X}\} \mathcal{U}\right) \mathcal{A}^{\prime} \check{\boldsymbol{\Pi}}^{-1} \boldsymbol{y}
$$

Therefore, this "optimal" choice of $\mathcal{R}$ results in an unregularized estimator that is simply a weighted backprojection followed by the application of a "matched" filter (convolution with $\overline{\boldsymbol{f}}_{s}$ ). This agrees with the conclusion from Section III-D6 that regularization is not essential for the $\mathrm{CHO}$, if the passbands of the $\mathrm{CHO}$ channels are flat, nonoverlapping and with internal noise covariance of the form (49).

Similarly to Section III-D6, a degenerate case occurs when the channel passbands are flat and the mean signal spectrum $\left\{X_{k}\right\}$, system spectrum $\left\{\lambda_{k}\right\}$, and object power spectrum $\left\{\nu_{k}\right\}$ are all constant over each channel's passband. Then, the first upper bound in (75) is achieved for any choice of regularization 
$\left\{\omega_{k}\right\}$ that is also constant over each passband, including $\omega_{k}=$ 0 . Once again, under the internal noise model (49), the choice of regularization is not important in this detection task if there is little within-passband variation of the mean signal spectrum, the system spectrum, the object power spectrum, and/or the channel response itself.

10) $\mathrm{CHO}$ and QPWLS With a Roughness Penalty: In the previous section, where we placed no constraints on the form of the regularizer $\mathcal{R}$, we ended up with an "optimal" $\mathcal{R}$ that yielded the unregularized estimator in (78). We now constrain the regularizer to the commonly used form of a quadratic roughness penalty. In this case, the regularization operator $\mathcal{R}$ in (14) is such that

$$
R(\boldsymbol{f})=\beta \sum_{j=1}^{n_{\mathrm{p}}} \sum_{k \in \mathcal{N}_{j}}\left(f_{j}-f_{k}\right)^{2}
$$

where $f_{j}$ is the $j$ th sample of the object $\boldsymbol{f}, \mathcal{N}_{j}$ a neighborhood of pixels around the $j$ th pixel, and $\beta \geq 0$ a regularization parameter. A regularization term of the form (79) penalizes differences between neighboring image pixels, thus favoring smoother images. For simplicity, we consider here a first-order neighborhood $\mathcal{N}_{j}$, consisting of the four closest (top, bottom, left and right) neighboring pixels. The only free parameter in the regularizer (79) is, thus, the regularization parameter $\beta$.

We now provide an example of how $\beta$ affects the SNR of the $\mathrm{CHO}$ with overlapping or nonoverlapping passbands in the presence of internal noise. We consider the case where $\mathcal{A}$ corresponds to a 2-D PET system model with the characteristics of a CTI ECAT 931 scanner (matrix size $128 \times 128$, pixel size $4.7 \mathrm{~mm}, 192$ radial samples with $3.1 \mathrm{~mm}$ spacing, 160 projection angles over $180^{\circ}$ ), generated by the ASPIRE software package [46]. We assume that the target signal $\boldsymbol{f}_{s}$ has a known Gaussian shape with full-width at half-maximum (FWHM) 4 pixels and amplitude 0.1 , the background $\boldsymbol{f}_{b}$ has a Gaussian autocorrelation function with FWHM 8 pixels and standard deviation 0.05 , and the mean background $\bar{f}_{b}$ is the anthropomorphic phantom shown in Fig. 2, which corresponds to a slice of the Zubal phantom [47]. We determine measurement variance by assuming a total of $5 \times 10^{5}$ counts.

We consider three different models for the CHO channels: Nonoverlapping square channels with $M=4$ (SQR) and overlapping difference-of-Gaussians channels with $M=3$ (S-DOG) and $M=10$ (D-DOG), as defined by Abbey et al. [16]. Fig. 3 shows plots of the SNR for QPWLS reconstruction with the roughness penalty in (79) and various values of $\beta$. The SNR is plotted for each of the three channel sets mentioned above and internal noise covariance

$$
\boldsymbol{\Pi}_{\mathrm{int}}=\sigma^{2} \boldsymbol{I}
$$

with $\sigma^{2}=0.005$. All the SNR values in these plots are normalized with respect to the ideal SNR in (26). The abscissa of the plots represents the resolution of the QPWLS reconstruction, defined as the FWHM (in pixels) of the reconstructed image when the true object is a noiseless impulse. This FWHM is a measure of the amount of smoothing imposed by QPWLS. The

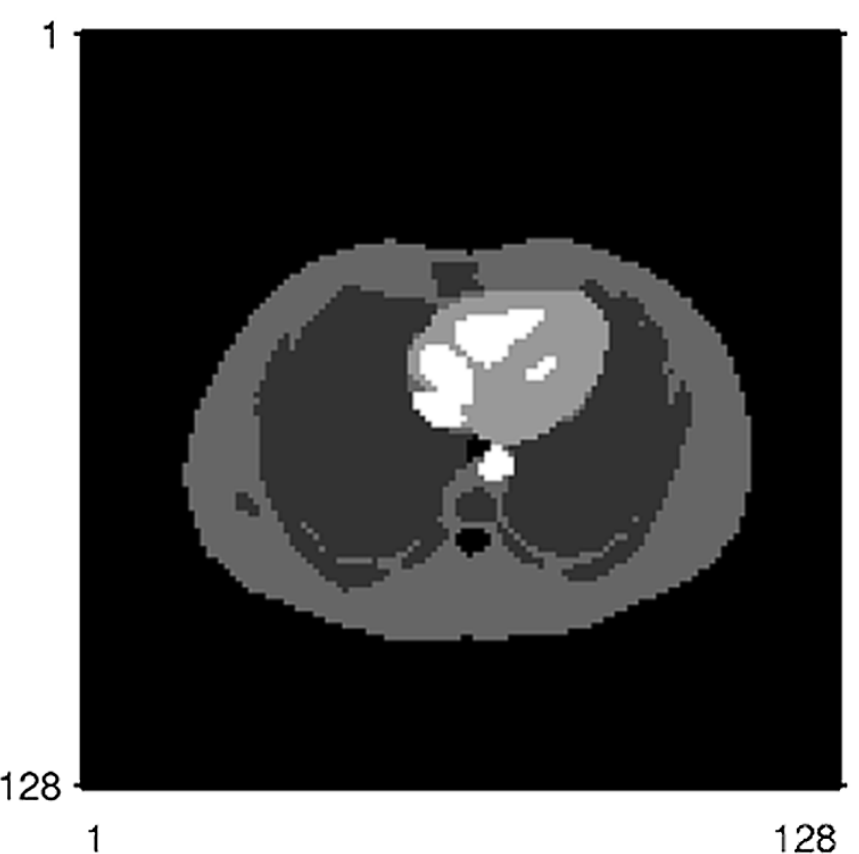

(a)

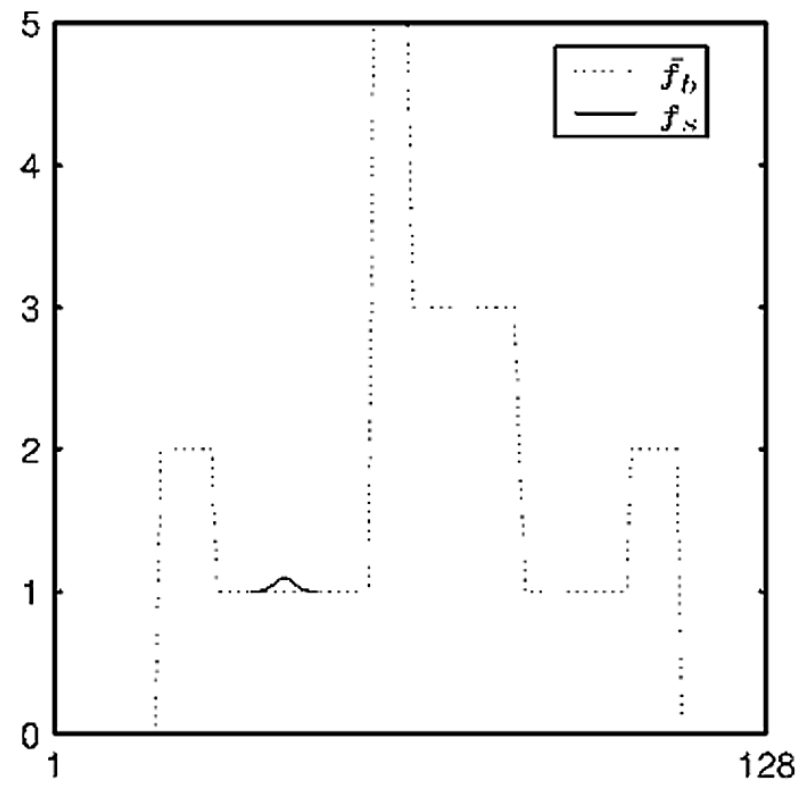

(b)

Fig. 2. Mean background and profile through the mean background with the target signal superimposed. (a) Mean background and (b) profile with signal.

resolution equals 1 pixel for $\beta=0$, which corresponds to unregularized WLS, and it increases as $\beta$ increases.

For all three channel sets, Fig. 3 shows a sharp SNR drop for very large amounts of regularization. Similar behavior was reported by Qi [24], who also shows that this SNR drop occurs only when internal observer noise is present. Apparently, internal noise is an important factor to consider when optimizing regularization methods with respect to detectability.

However, no similar drop in SNR occurs for very small amounts of regularization. In Fig. 3, for all three channel sets and with internal noise present, the peak SNR achieved by QPWLS is only slightly higher than the SNR achieved by 


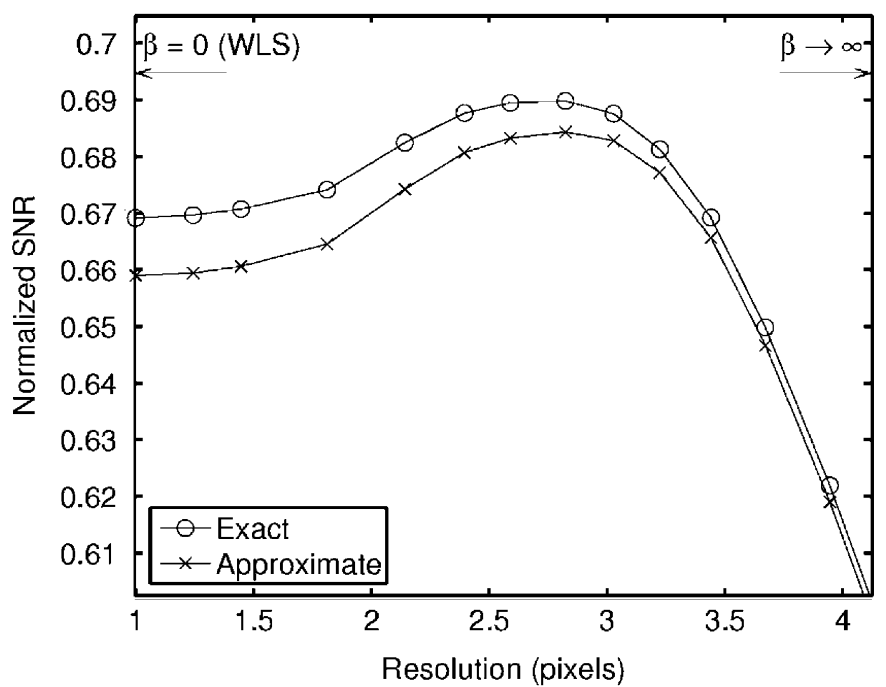

(a)

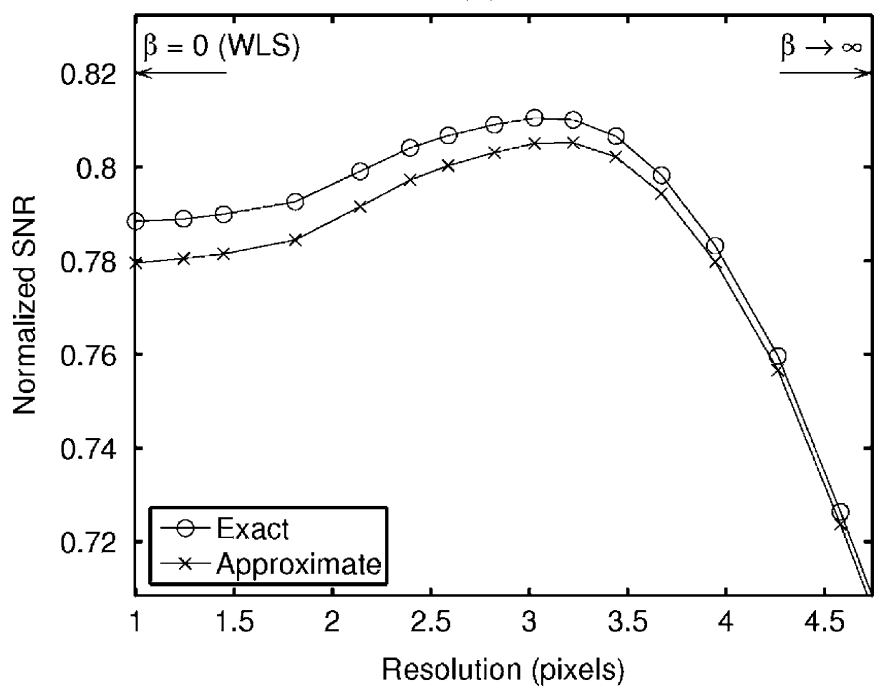

(b)

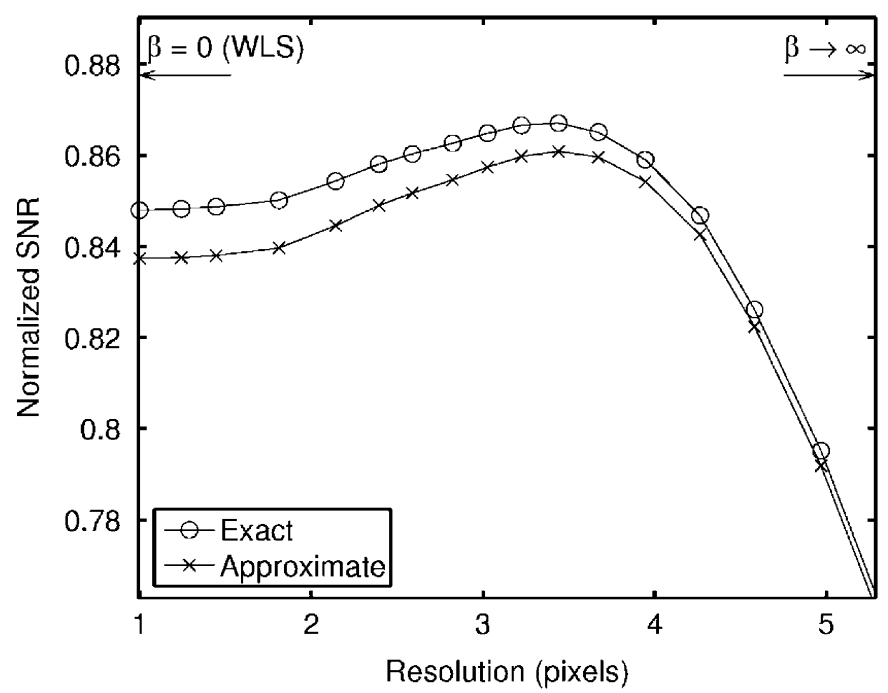

(c)

Fig. 3. SNR of $\mathrm{CHO}$ versus QPWLS reconstruction resolution for three different channel sets. The exact and approximate SNR is computed from (70) and (73), respectively. All SNR values are normalized with respect to the upper bound (ideal SNR for the internal-noise-free observer) in (26). (a) SQR channels, (b) S-DOG channels, and (c) D-DOG channels. unregularized WLS $(\beta=0)$. We also investigated different amounts of imaging noise and/or background variability and obtained very similar results (not shown here). That is, unregularized WLS led to SNR very close to the peak SNR attainable with QPWLS and the roughness penalty in (79). Once again, we have a situation in which regularization is of minimal benefit in the known-location detection task-even with a $\mathrm{CHO}$ model that includes overlapping channel passbands and internal noise.

Fig. 3 also compares the exact SNR, computed from (70), to the approximate SNR, computed from (73). The agreement between the two confirms the accuracy of our Fourier-domain approximations.

\section{CONCLUSION}

Our analysis shows that, for the task of known-location signal detection, there are unregularized reconstruction methods that allow several well-known observer models to achieve exactly the optimal SNR attainable with any combination of a linear observer and linear reconstructor. As mentioned in Section II-C, several popular reconstruction methods can be approximated as linear, especially around the signal location, where the nonnegativity constraint can be assumed inactive. It is plausible that a nonlinear reconstructor could improve the SNR. In the SKE tasks, however, the ideal linear SNR in (24), which can be achieved with unregularized reconstruction, $i s$ the overall ideal SNR and no nonlinear reconstruction method can improve it. This also holds approximately in the case of weak, spatially localized signals, whose shape variability can be assumed small in comparison to the background variability.

Even for the $\mathrm{CHO}$ with internal noise, there are situations where unregularized reconstruction leads to SNR very close to the peak SNR attainable with the given channels and internal noise level. For the proportional internal covariance model (49), we showed this analytically in Sections III-D6 and III-D9. For the constant internal covariance model (80), we presented an example in Section III-D10. The appropriate internal noise model would have to be determined from experiments with human observers. For the models considered here, we conclude that optimizing regularized reconstruction does not lead to any signifi-cant improvement of SNR performance in comparison to unregularized reconstruction in known-location detection tasks. This conclusion concurs with experimental results showing that the postsmoothing of noisy images has little benefit for human observers as far as performance in a known-location task is concerned [16].

It is interesting to contrast this to the results of Qi and Leahy, who analyzed the effect of regularization on the contrast-to noise ratio (CNR) [43]. As opposed to the SNR, the CNR exhibits a pronounced peak for an intermediate amount of regularization. This may be explained by the fact that the CNR is equivalent to the SNR of an observer that utilizes an impulse as its template. Since this template performs no smoothing of its own, the optimal reconstructor must perform some smoothing. On the other hand, the templates of the observers that we considered here are all weighted versions of the mean signal $\bar{f}_{s}$ (typically not an impulse), so they do perform some smoothing on the reconstructed image and, as a result, there is little benefit in terms of the SNR from additional smoothing performed by the reconstructor. 
The relatively small significance of regularization throughout our analysis indicates that detection performance in tasks where the target signal location is known exactly is of limited use as a criterion for optimizing the free parameters of regularized reconstruction methods. Furthermore, the finding that some form or another of unfiltered backprojection is an optimal reconstructor for several of the observer models considered here, shows that resolution is not an essential image quality as far as known-location detectability is concerned. Since known-location tasks are easy enough to perform optimally with poor resolution, it is important to examine whether there are more complicated tasks where regularization has a more significant effect. As discussed in detail in [48], regularization is more important for channelized observers that cannot prewhiten than it is for the CHO. Some preliminary experimental results indicate that humans may be less able to prewhiten in tasks where the location of the signal is unknown [49], [50]. The analysis of regularized image reconstruction methods with respect to unknown-location detectability is a promising direction that recent work is in the process of exploring [51]-[53].

\section{ACKNOWLEDGMENT}

The authors would like to thank the anonymous reviewers, whose careful reading and detailed comments improved the paper substantially.

\section{REFERENCES}

[1] H. H. Barrett, "Objective assessment of image quality: Effects of quantum noise and object variability," J. Opt. Soc. Am. A, vol. 7, no. 7, pp. 1266-1278, Jul. 1990.

[2] L. B. Lusted, "Signal detectability and medical decision-making," Science, vol. 171, no. 3977, pp. 1217-19, Mar. 1971.

[3] C. E. Metz, "Basic principles of ROC analysis," Sem. Nucl. Med., vol. 8, no. 4, pp. 283-98, Oct. 1978.

[4] M. S. Chesters, "Human visual perception and ROC methodology in medical imaging," Phys. Med. Biol., vol. 37, no. 7, pp. 1433-84, Jul. 1992.

[5] H. L. Van Trees, Detection, Estimation, and Modulation Theory. New York: Wiley, 1968.

[6] A. E. Burgess, R. F. Wagner, R. J. Jennings, and H. B. Barlow, "Efficiency of human visual signal discrimination," Science, vol. 214, no. 4516, pp. 93-94, Oct. 1981.

[7] L. N. D. Loo, K. Doi, and C. E. Metz, "A comparison of physical image comparison indexes and observer performance in the radiographic detection of nylon beads," Phys. Med. Biol., vol. 29, no. 7, pp. 837-57, Jul. 1984.

[8] A. E. Burgess and B. Colborne, "Visual signal detection. IV. Observer inconsistency," J. Opt. Soc. Am. A, vol. 5, no. 4, p. 617, Apr. 1988.

[9] K. J. Myers and H. H. Barrett, "Addition of a channel mechanism to the ideal-observer model," J. Opt. Soc. Am. A, vol. 4, no. 12, pp. 2447-57, Dec. 1987.

[10] Handbook of Medical Imaging, Volume 1. Physics and Psychophysics, SPIE, Bellingham, Washington, 2000, pp. 558-92. K. J. Myers, Ideal observer models of visual signal detection, J. Beutel, H. L. Kundel, and R. L. Van Metter Eds.

[11] H. H. Barrett and K. J. Myers, Foundations of Image Science. New York: Wiley, 2003.

[12] J. P. Rolland and H. H. Barrett, "Effect of random background inhomogeneity on observer detection performance," J. Opt. Soc. Am. A, vol. 9, no. 5, pp. 649-58, May 1992.

[13] C. K. Abbey and H. H. Barrett, "Linear iterative reconstruction algorithms: Study of observer performance," in Information Processing in Medical Im., Y. Bizais, C. Barillot, and R. D. Paola, Eds. Dordrect, The Netherlands: Kluwer, 1995, pp. 65-76.
[14] S. D. Wollenweber, B. M. W. Tsui, D. S. Lalush, E. C. Frey, K. J. LaCroix, and G. T. Gullberg, "Comparison of Hotelling observer models and human observers in defect detection from myocardial SPECT imaging," IEEE Trans. Nucl. Sci., vol. 46, no. 6, pp. 2098-103, Dec. 1999.

[15] H. C. Gifford, M. A. King, D. J. de Vries, and E. J. Soares, "Channelized Hotelling and human observer correlation for lesion detection in hepatic SPECT imaging," J. Nucl. Med., vol. 41, no. 3, pp. 514-21, Mar. 2000.

[16] C. K. Abbey and H. H. Barrett, "Human- and model-observer performance in ramp-spectrum noise: Effects of regularization and object variability," J. Opt. Soc. Am. A, vol. 18, no. 3, pp. 473-88, Mar. 2001.

[17] S. Sankaran, E. C. Frey, K. L. Gilland, and B. M. Tsui, "Optimum compensation method and filter cutoff frequency in myocardial spect: A human observer study," J. Nucl. Med., vol. 43, no. 3, pp. 432-8, Mar. 2002.

[18] C. K. Abbey and M. P. Eckstein, "Maximum-likelihood and maximum-a-posteriori estimates of human-observer templates," Proc. SPIE (Medical Imaging 2001: Image Perception Performance)., vol. 4324, pp. 114-22, 2001

[19] — "Optimal shifted estimates of human-observer templates in twoalternative forced-choice experiments," IEEE Trans. Med. Imag., vol. 21, no. 5, pp. 429-40, May 2002.

[20] P. Bonetto, J. Qi, and R. M. Leahy, "Covariance approximation for fast and accurate computation of channelized Hotelling observer statistics," IEEE Trans. Nucl. Sci., vol. 47, no. 4, pp. 1567-72, Aug. 2000.

[21] J. Qi and R. H. Huesman, "Theoretical study of lesion detectability of MAP reconstruction using computer observers," IEEE Trans. Med. Imag., vol. 20, no. 8, pp. 815-22, Aug. 2001.

[22] J. A. Fessler and A. Yendiki, "Channelized Hotelling observer performance for penalized-likelihood image reconstruction," in Proc. IEEE Nuclear Science Symp. Medical Imaging Conf., vol. 2, 2002, pp. 1040-4.

[23] Y. Xing, I.-T. Hsiao, and G. Gindi, "Rapid calculation of detectability in Bayesian single photon emission computed tomography," Phys. Med. Biol., vol. 48, no. 22, pp. 3755-3774, Nov. 2003.

[24] J. Qi, "Analysis of lesion detectability in Bayesian emission reconstruction with nonstationary object variability," IEEE Trans. Med. Imag., vol. 23, no. 3, pp. 321-9, Mar. 2004.

[25] A. Yendiki and J. A. Fessler, "Analytical approach to channelized Hotelling observer performance for regularized tomographic image reconstruction," in Proc. IEEE Int. Symp. Biomedical Imaging, 2004, pp. $360-363$.

[26] J. Qi, "Comparison of statistical reconstructions with isotropic and anisotropic resolution," in Proc. IEEE Nuclear Science Symp. Medical Imaging Conf., vol. 6, 2004, pp. 3624-3628.

[27] T. Lewellen and J. Carp, "PET systems," in Emission Tomography, M. N. Wernick and J. N. Aarsvold, Eds. San Diego, CA: Academic, 2004, ch. 10

[28] M. A. King, S. J. Glick, P. H. Pretorius, R. G. Wells, H. C. Gifford, M. V. Narayanan, and T. Farncombe, "Attenuation, scatter, and spatial resolution compensation in SPECT," in Emission Tomography, M. N. Wernick and J. N. Aarsvold, Eds. San Diego, CA: Academic, 2004, ch. 22.

[29] J. A. Fessler, "Mean and variance of implicitly defined biased estimators (such as penalized maximum likelihood): Applications to tomography," in IEEE Trans. Image Process., vol. 5, Mar. 1996, pp. 493-506.

[30] K. Fukunaga, Introduction to Statistical Pattern Recognition. Boston, MA: Academic, 1990.

[31] H. H. Barrett, T. Gooley, K. Girodias, J. Rolland, T. White, and J. Yao, "Linear discriminants and image quality," in Information Processing in Medical Im., A. C. F. Colchester and D. J. Hawkes, Eds. Berlin, Germany: Springer-Verlag, 1991, pp. 458-73.

[32] H. H. Barrett, J. Yao, J. P. Rolland, and K. J. Myers, "Model observers for assessment of image quality," Proc. Nat. Acad. Sci., vol. 90, pp. 9758-65, Nov. 1993.

[33] J. Qi and R. M. Leahy, "Resolution and noise properties of MAP reconstruction for fully 3-D PET,' IEEE Trans. Med. Imag., vol. 19, no. 5, pp. 493-506, May 2000.

[34] R. O. Duda, P. E. Hart, and D. G. Stork, Pattern classification. New York, NY: Wiley, 2001.

[35] H. V. Henderson and S. R. Searle, "On deriving the inverse of a sum of matrices," SIAM Rev., vol. 23, no. 1, pp. 53-60, Jan. 1981.

[36] F. A. Graybill, Matrices with Applications in Statistics, 2nd ed. Bellmont, CA: Wadsworth, 1983.

[37] R. F. Wagner, D. G. Brown, and C. E. Metz, "On the multiplex advantage of coded source/aperture photon imaging," Proc. SPIE (Digital Radiography), vol. 314, pp. 72-76, 1981. 
[38] K. J. Myers, J. P. Rolland, H. H. Barrett, and R. F. Wagner, "Aperture optimization for emission imaging: Effect of a spatially varying background," J. Opt. Soc. Am. A, vol. 7, no. 7, pp. 1279-93, Jul. 1990.

[39] B. D. Gallas and H. H. Barrett, "Validating the use of channels to estimate the ideal linear observer," J. Opt. Soc. Am. A, vol. 20, no. 9, pp. 1725-38, Sep. 2003.

[40] H. C. Gifford, R. G. Wells, and M. A. King, "A comparison of human observer LROC and numerical observer ROC for tumor detection in SPECT images," IEEE Trans. Nucl. Sci., vol. 46, no. 4, pp. 1032-7, Aug. 1999.

[41] J. A. Fessler and W. L. Rogers, "Spatial resolution properties of penalized-likelihood image reconstruction methods: Space-invariant tomographs," IEEE Trans. Image Process., vol. 5, no. 9, pp. 1346-58, Sep. 1996.

[42] J. A. Fessler and S. D. Booth, "Conjugate-gradient preconditioning methods for shift-variant PET image reconstruction," in IEEE Trans. Image Process., vol. 8, May 1999, pp. 688-99.

[43] J. Qi and R. M. Leahy, "A theoretical study of the contrast recovery and variance of MAP reconstructions with applications to the selection of smoothing parameters," IEEE Trans. Med. Imag., vol. 18, no. 4, pp. 293-305, Apr. 1999.

[44] J. W. Stayman and J. A. Fessler, "Regularization for uniform spatial resolution properties in penalized-likelihood image reconstruction," IEEE Trans. Med. Imag., vol. 19, no. 6, pp. 601-15, Jun. 2000.

[45] J. A. Fessler, "Analytical approach to regularization design for isotropic spatial resolution," in Proc. IEEE Nuclear Science Symp. Medical Imaging Conf., vol. 3, 2003, pp. 2022-2026.

[46] — - (1995, Jul.) ASPIRE 3.0 User's Guide: A Sparse Iterative Reconstruction Library. Commun. Signal Process. Lab., Dept. EECS, Univ. Michigan, Ann Arbor. [Online]. Available: http://www.eecs.umich.edu/ fessler
[47] G. Zubal, G. Gindi, M. Lee, C. Harrell, and E. Smith, "High resolution anthropomorphic phantom for Monte Carlo analysis of internal radiation sources," in Proc. IEEE Symp. Computer-Based Medical Systems, 1990 pp. 540-547.

[48] A. Yendiki, "Analysis of Signal Detectability in Statistically Reconstructed Tomorgaphic Images," Ph.D. dissertation, Elect. Eng.: Syst., Univ. Michigan, Ann Arbor, 2005.

[49] H. C. Gifford, P. H. Pretorius, and M. A. King, "Comparison of humanand model-observer LROC studies," Proc. SPIE (Medical Imaging 2003: Image Perception, Observer Performance, and Technology Assessment), vol. 5034, pp. 112-122, 2003.

[50] H. C. Gifford, P. E. Kinahan, C. Lartizien, and M. A. King, "Evaluation of multiclass model observers in PET LROC studies," in Proc. IEEE Nuclear Science Symp. Medical Imaging Conf., vol. 7, 2004, pp. 4068--4071.

[51] P. K. Khurd and G. R. Gindi, "LROC model observers for emission tomographic reconstruction," Proc. SPIE (Medical Imaging 2004: Image Perception, Observer Performance, and Technology Assessment), vol. 5372, pp. 509-520, 2004.

[52] J. Qi and R. H. Huesman, "Fast approach to evaluate MAP reconstruction for lesion detection and localization," in Proc. SPIE (Medical Imaging 2004: Image Perception, Observer Performance, and Technology Assessment), vol. 5372, 2004, pp. 273-282.

[53] A. Yendiki and J. A. Fessler, "Analysis of observer performance in detecting signals with location uncertainty for regularized tomographic image reconstruction," in Proc. IEEE Nuclear Science Symp. Medical Imaging Conf., vol. 4, 2004, pp. 2620-2624. 